\title{
Solving the Conformal Constraints for Scalar Operators in Momentum Space and the Evaluation of Feynman's Master Integrals
}

\author{
${ }^{a}$ Claudio Corianò, ${ }^{a}$ Luigi Delle Rose, ${ }^{b}$ Emil Mottola and ${ }^{a}$ Mirko Serino \\ a Dipartimento di Matematica e Fisica "Ennio De Giorgi", \\ Università del Salento \\ and \\ INFN Lecce, Via Arnesano 73100 Lecce, Italy \\ b Theoretical Division, Los Alamos National Laboratory \\ Los Alamos, NM, 87545, USA*
}

\begin{abstract}
We investigate the structure of the constraints on three-point correlation functions emerging when conformal invariance is imposed in momentum space and in arbitrary space-time dimensions, presenting a derivation of their solutions for arbitrary scalar operators. We show that the differential equations generated by the requirement of symmetry under special conformal transformations coincide with those satisfied by generalized hypergeometric functions (Appell's functions). Combined with the position space expression of this correlator, whose Fourier transform is given by a family of generalized Feynman (master) integrals, the method allows to derive the expression of such integrals in a completely independent way, bypassing the use of Mellin-Barnes techniques, which have been used in the past. The application of the special conformal constraints generates a new recursion relation for this family of integrals.
\end{abstract}

*claudio.coriano@unisalento.it, luigi.dellerose@le.infn.it,emil@lanl.gov,mirko.serino@le.infn.it 


\section{Introduction}

Conformal invariance plays an important role in constraining the structure of correlation functions of conformal field theories in any dimensions. It allows to fix the form of correlators - up to three-point functions - modulo a set of constants which are also given, once the field content of the underlying conformal field theory is selected [1, 2]. The approach, which is largely followed in this case, is naturally formulated in position space, while the same conformal requirements, in momentum space, have been far less explored [3].

Conformal three-point functions have been intensively studied in the past, and a classification of their possible structures, in the presence of conformal anomalies, is available. Conformal anomalies emerge due to the inclusion of the energy momentum tensor in a certain correlator and, in some cases, find specific realizations in free field theories of scalars, vectors and fermions 1, 2. Typical correlators which have been studied are those involving the TT, TOO, TVV , and $T T T$, where $T$ denotes the energy momentum tensor, $V$ a vector and $O$ a generic scalar operator of arbitrary dimension.

The conformal constraints in position space, in this case, are combined with the Ward identities derived from the conservation of the energy momentum tensor and its tracelessness condition, valid at separate coordinate points, to fix the structure of each correlator. These solutions are obtained for generic conformal theories, with no reference to their Lagrangian realization which, in general, may not even exist. The solutions of the conformal constraints are then extended to include the contributions from the coincidence regions, where all the external points collapse to the same point.

Free field theory realizations of these correlators (for fermions, scalars and vectors) allow to

perform a direct test of these results both in position and in momentum space, at least in some important cases, such as the $T V V$ or the TTT (this latter only for $d=4$ ) [3], but obviously do not exhaust all possibilities.

Recently interest in the momentum space form of conformal correlators has arisen in the context of the study of anomalous conformal Ward identities, massless poles and scalar degrees of freedom associated with the trace anomaly [4, 5, 6, 7, 8, 9, 10], and because of their possible role in determining the form of conformal invariance in the non-Gaussian features of the Cosmic Microwave Background [11, 12], or in inflation [13, 14]. The possibility of retrieving information on conformal correlators in momentum space seems to be related, in one way or another, to the previous knowledge of the same correlators in configuration space, where the conformal constraints are easier to implement and solve. One question that can be naturally raised is if we are able to bypass the study of conformal correlators in position space, by fixing their structure directly in momentum space and with no further input. This approach defines an independent 
path which, as we are going to show, can be successful in some specific cases. We will illustrate the direct construction of the solution bringing the example of the scalar three-point correlator. The analysis in momentum space should not be viewed though as an unnecessary complication. In fact, the solution in the same space, if found, is explicit in the momentum variables and can be immediately compared with the integral representation of the position space solution, given by a generalized Feynman integral. As a corollary of this approach, in the case of threepoint functions, we are able to determine the complete structure of such an integral, which is characterized by three free parameters related to the scaling dimensions of the original scalar operators, in an entirely new way. It is therefore obvious that this approach allows to determine the explicit form of an entire family of master integrals.

In the scalar case we are able to show that the conformal conditions are equivalent to partial differential equations (PDE's) of generalized hypergeometric type, solved by functions of two variables, $x$ and $y$, which take the form of ratios of the external momenta. The general solution is expressed as a generic linear combination of four generalized hypergeometric functions of the same variables, or Appell's functions. Three out of the four constants of the linear combination can be fixed by the momentum symmetry. This allows to write down the general form of the scalar correlator in terms of a single multiplicative constant, which classifies all the possible conformal realizations of the scalar three-point function.

In the final part of this work we go back to the analysis of the conformal master integrals, the Fourier transform of the scalar three-point correlators in position space. We show that the usual rules of integration by parts satisfied by these integrals are nothing else but the requirement of scale invariance. Specifically, dilatation symmetry relates the master integral $J\left(\nu_{1}, \nu_{2}, \nu_{3}\right)$, labelled by the powers of the Feynman propagators $\left(\nu_{1}, \nu_{2}, \nu_{3}\right)$ - with $\nu_{1}+\nu_{2}+\nu_{3}=\kappa$ - to those of the first neighboring plane $(\kappa \rightarrow \kappa+1)$. On the other hand, special conformal constraints relate the integrals of second neighboring planes $(\kappa \rightarrow \kappa+2)$.

\section{Conformal transformations}

In order to render our treatment self-contained, we present a brief review of the conformal transformations in $d>2$ dimensions which identify, in Minkowski space, the conformal group $S O(2, d)$.

These may be defined as the transformations $x_{\mu} \rightarrow x_{\mu}^{\prime}(x)$ that preserve the infinitesimal length up to a local factor

$$
d x_{\mu} d x^{\mu} \rightarrow d x_{\mu}^{\prime} d x^{\prime \mu}=\Omega(x)^{-2} d x_{\mu} d x^{\mu}
$$


In the infinitesimal form, for $d>2$, the conformal transformations are given by

$$
x_{\mu}^{\prime}(x)=x_{\mu}+a_{\mu}+\omega_{\mu}{ }^{\nu} x_{\nu}+\lambda x_{\mu}+b_{\mu} x^{2}-2 x_{\mu} b \cdot x
$$

with

$$
\Omega(x)=1-\sigma(x) \quad \text { and } \quad \sigma(x)=\lambda-2 b \cdot x .
$$

The transformation in Eq.(2) is defined by translations $\left(a_{\mu}\right)$, rotations $\left(\omega_{\mu \nu}=-\omega_{\nu \mu}\right)$, dilatations $(\lambda)$ and special conformal transformations $\left(b_{\mu}\right)$. The first two define the Poincaré subgroup which leaves invariant the infinitesimal length $(\Omega(x)=1)$.

If we also consider the inversion

$$
x_{\mu} \rightarrow x_{\mu}^{\prime}=\frac{x_{\mu}}{x^{2}}, \quad \Omega(x)=x^{2},
$$

we can enlarge the conformal group to $O(2, d)$. Special conformal transformations can be realized by a translation preceded and followed by an inversion.

Having specified the elements of the conformal group, we can define a quasi primary field $\mathcal{O}^{i}(x)$, where the index $i$ runs over a representation of the group $O(1, d-1)$ to which the field belongs, through the transformation property under a conformal transformation $g$

$$
\mathcal{O}^{i}(x) \stackrel{g}{\rightarrow} \mathcal{O}^{i}\left(x^{\prime}\right)=\Omega(x)^{\eta} D_{j}^{i}(g) \mathcal{O}^{j}(x),
$$

where $\eta$ is the scaling dimension of the field and $D_{j}^{i}(g)$ denotes the representation of $O(1, d-1)$. In the infinitesimal form we have

$$
\delta_{g} \mathcal{O}^{i}(x)=-\left(L_{g} \mathcal{O}\right)^{i}(x), \quad \text { with } \quad L_{g}=v \cdot \partial+\eta \sigma+\frac{1}{2} \partial_{[\mu} v_{\nu]} \Sigma^{\mu \nu},
$$

where the vector $v_{\mu}$ is the infinitesimal coordinate variation $v_{\mu}=\delta_{g} x_{\mu}=x_{\mu}^{\prime}(x)-x_{\mu}$ and $\left(\Sigma_{\mu \nu}\right)_{j}^{i}$ are the generators of $O(1, d-1)$ in the representation of the field $\mathcal{O}^{i}$. The explicit form of the operator $L_{g}$ can be obtained from Eq.(2) and Eq.(3) and is given by

$$
\begin{array}{llrl} 
& \text { translations: } & L_{g} & =a^{\mu} \partial_{\mu}, \\
& \text { rotations: } & L_{g} & =\frac{\omega^{\mu \nu}}{2}\left[x_{\nu} \partial_{\mu}-x_{\mu} \partial_{\nu}-\Sigma_{\mu \nu}\right], \\
& \text { scale transformations : } & L_{g} & =\lambda[x \cdot \partial+\eta], \\
\text { special conformal transformations. : } & L_{g} & =b^{\mu}\left[x^{2} \partial_{\mu}-2 x_{\mu} x \cdot \partial-2 \eta x_{\mu}-2 x_{\nu} \Sigma_{\mu}{ }^{\nu}\right] .
\end{array}
$$

Conformal invariant correlation functions of quasi primary fields can be defined by requiring that

$$
\sum_{r=1}^{n}\left\langle\mathcal{O}_{1}^{i_{1}}\left(x_{1}\right) \ldots \delta_{g} \mathcal{O}_{r}^{i_{r}}\left(x_{r}\right) \ldots \mathcal{O}_{n}^{i_{n}}\left(x_{n}\right)\right\rangle=0
$$


In particular, the invariance under scale and special conformal transformations, in which we are mainly interested, reads as

$$
\begin{aligned}
& \sum_{r=1}^{n}\left(x_{r} \cdot \partial^{x_{r}}+\eta_{r}\right)\left\langle\mathcal{O}_{1}^{i_{1}}\left(x_{1}\right) \ldots \mathcal{O}_{r}^{i_{r}}\left(x_{r}\right) \ldots \mathcal{O}_{n}^{i_{n}}\left(x_{n}\right)\right\rangle=0, \\
& \sum_{r=1}^{n}\left(x_{r}^{2} \partial_{\mu}^{x_{r}}-2 x_{r \mu} x_{r} \cdot \partial^{x_{r}}-2 \eta_{r} x_{r \mu}-2 x_{r \nu}\left(\Sigma_{\mu}^{(r)^{\nu}}\right)_{j_{r}}^{i_{r}}\right)\left\langle\mathcal{O}_{1}^{i_{1}}\left(x_{1}\right) \ldots \mathcal{O}_{r}^{j_{r}}\left(x_{r}\right) \ldots \mathcal{O}_{n}^{i_{n}}\left(x_{n}\right)\right\rangle=0 .
\end{aligned}
$$

The constraints provided by conformal invariance have been solved in coordinate space and for arbitrary space-time dimension. One can show, for instance, that the two and three-point functions are completely determined by conformal symmetry up to a small number of independent constants [15, 1].

Exploiting the same constraints in momentum space is somewhat more involved. In the following we assume invariance under the Poincaré group and we focus our attention on dilatations and special conformal transformations.

For this purpose we define the Fourier transform of a $n$ point correlation function as

$$
\begin{aligned}
(2 \pi)^{d} \delta^{(d)}\left(p_{1}\right. & \left.+\ldots+p_{n}\right)\left\langle\mathcal{O}_{1}^{i_{1}}\left(p_{1}\right) \ldots \mathcal{O}_{n}^{i_{n}}\left(p_{n}\right)\right\rangle \\
& =\int d^{d} x_{1} \ldots d^{d} x_{n}\left\langle\mathcal{O}_{1}^{i_{1}}\left(x_{1}\right) \ldots \mathcal{O}_{n}^{i_{n}}\left(x_{n}\right)\right\rangle e^{i p_{1} \cdot x_{1}+\ldots+i p_{n} \cdot x_{n}}
\end{aligned}
$$

where the correlation function in momentum space is understood to depend only on $n-1$ momenta, as the $n$-th one is removed using momentum conservation.

The momentum space differential equations describing the invariance under dilatations and special conformal transformations are obtained Fourier-transforming Eq.(9). It is worth noting that some care must be taken, due to the appearance of derivatives on the delta function. As pointed out in [13], these terms can be discarded and we are left with the two equations

$$
\begin{gathered}
{\left[-\sum_{r=1}^{n-1}\left(p_{r \mu} \frac{\partial}{\partial p_{r \mu}}+d\right)+\sum_{r=1}^{n} \eta_{r}\right]\left\langle\mathcal{O}_{1}^{i_{1}}\left(p_{1}\right) \ldots \mathcal{O}_{r}^{i_{r}}\left(p_{r}\right) \ldots \mathcal{O}_{n}^{i_{n}}\left(p_{n}\right)\right\rangle=0,} \\
\sum_{r=1}^{n-1}\left(p_{r \mu} \frac{\partial^{2}}{\partial p_{r}^{\nu} \partial p_{r \nu}}-2 p_{r \nu} \frac{\partial^{2}}{\partial p_{r}^{\mu} \partial p_{r \nu}}+2\left(\eta_{r}-d\right) \frac{\partial}{\partial p_{r}^{\mu}}+2\left(\Sigma_{\mu \nu}^{(r)}\right)_{j_{r}}^{i_{r}} \frac{\partial}{\partial p_{r \nu}}\right) \\
\times\left\langle\mathcal{O}_{1}^{i_{1}}\left(p_{1}\right) \ldots \mathcal{O}_{r}^{j_{r}}\left(p_{r}\right) \ldots \mathcal{O}_{n}^{i_{n}}\left(p_{n}\right)\right\rangle=0,
\end{gathered}
$$

which define an arbitrary conformal invariant correlation function in $d$ dimensions. Note that we are dealing with a first and a second order partial differential equations in $n-1$ independent momenta. The choice of the momentum which is eliminated in the two equations given above 
is arbitrary.

Despite the apparent asymmetry in the definition of the special conformal constraint, due to the absence of the $n$-th scaling dimension $\eta_{n}$ and the $n$-th spin matrix $\left(\Sigma_{\mu \nu}^{(n)}\right)_{j_{n}}^{i_{n}}$, the second of Eq.(11) does not depend on the specific momentum which is eliminated. We could have similarly chosen to express Eq.(11) in terms of the momenta $\left(p_{1} \ldots p_{k-1}, p_{k+1}, \ldots p_{n}\right)$, with $p_{k}$ removed using the momentum conservation, and we would have obtained an equivalent relation. We have left to an appendix the formal proof of this point. We have then explicitly verified the correctness of our assertion on the vector and tensor two-point correlators which have been discussed in the following section. In both cases, whatever momentum parameterization is chosen for Eq.(11), we have checked that special conformal constraints imply the equality of the scaling dimensions of the two (vector or tensor) operators, accordingly to the well-known result obtained in coordinate space.

We recall, anyway, that, apart from the following section, in which vector and tensor two-point functions are reviewed, the main results of this work, being focused on scalar operators, are free from all the complications arising from the presence of the spin matrices in the special conformal constraints.

\section{Two-point functions from momentum space and anoma- lies}

\subsection{General solutions of the scale and special conformal identities}

We start exploring the implications of these constraints on two-point functions. In particular, the quasi primary fields taken into account are scalar $(\mathcal{O})$, conserved vector $\left(V_{\mu}\right)$ and conserved and traceless $\left(T_{\mu \nu}\right)$ operators.

For the two-point functions the differential equations in Eq.(11) simplify considerably, being expressed in terms of just one independent momentum $p$, and take the form

$$
\begin{aligned}
& \left(-p_{\mu} \frac{\partial}{\partial p_{\mu}}+\eta_{1}+\eta_{2}-d\right) G^{i j}(p)=0 \\
& \left(p_{\mu} \frac{\partial^{2}}{\partial p^{\nu} \partial p_{\nu}}-2 p_{\nu} \frac{\partial^{2}}{\partial p^{\mu} \partial p_{\nu}}+2\left(\eta_{1}-d\right) \frac{\partial}{\partial p^{\mu}}+2\left(\Sigma_{\mu \nu}\right)_{k}^{i} \frac{\partial}{\partial p_{\nu}}\right) G^{k j}(p)=0
\end{aligned}
$$

where we have defined $G^{i j}(p) \equiv\left\langle\mathcal{O}_{1}^{i}(p) \mathcal{O}_{2}^{j}(-p)\right\rangle$. The first of Eq.(12) dictates the scaling behavior of the correlation function, while special conformal invariance allows a non zero result only for equal scale dimensions of the two operators $\eta_{1}=\eta_{2}$, as we know from the corresponding analysis in coordinate space. We start by illustrating this point. 
For the correlation function $G_{S}(p)$ of two scalar quasi primary fields the invariance under the Poincaré group obviously implies that $G_{S}(p) \equiv G_{S}\left(p^{2}\right)$, so that the derivatives with respect to the momentum $p_{\mu}$ can be easily recast in terms of the variable $p^{2}$.

The invariance under scale transformations implies that $G_{S}\left(p^{2}\right)$ is a homogeneous function of degree $\alpha=\frac{1}{2}\left(\eta_{1}+\eta_{2}-d\right)$. At the same time, it is easy to show that the second equation in (12) can be satisfied only if $\eta_{1}=\eta_{2}$. Therefore conformal symmetry fixes the structure of the scalar two-point function up to an arbitrary overall constant $C$ as

$$
G_{S}\left(p^{2}\right)=\left\langle\mathcal{O}_{1}(p) \mathcal{O}_{2}(-p)\right\rangle=\delta_{\eta_{1} \eta_{2}} C\left(p^{2}\right)^{\eta_{1}-d / 2}
$$

If we redefine

$$
C=c_{S 12} \frac{\pi^{d / 2}}{4^{\eta_{1}-d / 2}} \frac{\Gamma\left(d / 2-\eta_{1}\right)}{\Gamma\left(\eta_{1}\right)}
$$

in terms of the new integration constant $c_{S 12}$, the two-point function reads as

$$
G_{S}\left(p^{2}\right)=\delta_{\eta_{1} \eta_{2}} c_{S 12} \frac{\pi^{d / 2}}{4^{\eta_{1}-d / 2}} \frac{\Gamma\left(d / 2-\eta_{1}\right)}{\Gamma\left(\eta_{1}\right)}\left(p^{2}\right)^{\eta_{1}-d / 2},
$$

and after a Fourier transformation in coordinate space takes the familiar form

$$
\left\langle\mathcal{O}_{1}\left(x_{1}\right) \mathcal{O}_{2}\left(x_{2}\right)\right\rangle \equiv \mathcal{F} \cdot \mathcal{T} \cdot\left[G_{S}\left(p^{2}\right)\right]=\delta_{\eta_{1} \eta_{2}} c_{S 12} \frac{1}{\left(x_{12}^{2}\right)^{\eta_{1}}},
$$

where $x_{12}=x_{1}-x_{2}$. The ratio of the two Gamma functions relating the two integration constants $C$ and $c_{S 12}$ correctly reproduces the ultraviolet singular behavior of the correlation function and plays a role in the discussion of the origin of the scale anomaly.

Now we turn to the vector case where we define $G_{V}^{\alpha \beta}(p) \equiv\left\langle V_{1}^{\alpha}(p) V_{2}^{\beta}(-p)\right\rangle$. If the vector current is conserved, then the tensor structure of the two-point correlation function is entirely fixed by the transversality condition, $\partial^{\mu} V_{\mu}=0$, as

$$
G_{V}^{\alpha \beta}(p)=\pi^{\alpha \beta}(p) f_{V}\left(p^{2}\right), \quad \text { with } \quad \pi^{\alpha \beta}(p)=\eta^{\alpha \beta}-\frac{p^{\alpha} p^{\beta}}{p^{2}}
$$

where $f_{V}$ is a function of the invariant square $p^{2}$ whose form, as in the scalar case, is determined by the conformal constraints. Following the same reasonings discussed previously we find that

$$
G_{V}^{\alpha \beta}(p)=\delta_{\eta_{1} \eta_{2}} c_{V 12} \frac{\pi^{d / 2}}{4^{\eta_{1}-d / 2}} \frac{\Gamma\left(d / 2-\eta_{1}\right)}{\Gamma\left(\eta_{1}\right)}\left(\eta^{\alpha \beta}-\frac{p^{\alpha} p^{\beta}}{p^{2}}\right)\left(p^{2}\right)^{\eta_{1}-d / 2},
$$

with $c_{V 12}$ being an arbitrary constant. We recall that the second equation in (12) gives consistent results for the two-point function in Eq.(18) only when the scale dimension $\eta_{1}=d-1$. 
We refer to appendix $\mathrm{B}$ for more details.

To complete this short excursus, we present the solution of the conformal constraints for the two-point function built out of two energy momentum tensor operators which are symmetric, conserved and traceless

$$
T_{\mu \nu}=T_{\nu \mu}, \quad \partial^{\mu} T_{\mu \nu}=0, \quad T_{\mu}{ }^{\mu}=0
$$

Exploiting the conditions defined in Eq.(19) we can unambiguously define the tensor structure of the correlation function $G_{T}^{\alpha \beta \mu \nu}(p)=\Pi_{d}^{\alpha \beta \mu \nu}(p) f_{T}\left(p^{2}\right)$ with

$$
\Pi_{d}^{\alpha \beta \mu \nu}(p)=\frac{1}{2}\left[\pi^{\alpha \mu}(p) \pi^{\beta \nu}(p)+\pi^{\alpha \nu}(p) \pi^{\beta \mu}(p)\right]-\frac{1}{d-1} \pi^{\alpha \beta}(p) \pi^{\mu \nu}(p),
$$

and the scalar function $f_{T}\left(p^{2}\right)$ determined as usual, up to a multiplicative constant, by requiring the invariance under dilatations and special conformal transformations. We obtain

$$
G_{T}^{\alpha \beta \mu \nu}(p)=\delta_{\eta_{1} \eta_{2}} c_{T 12} \frac{\pi^{d / 2}}{4^{\eta_{1}-d / 2}} \frac{\Gamma\left(d / 2-\eta_{1}\right)}{\Gamma\left(\eta_{1}\right)} \Pi_{d}^{\alpha \beta \mu \nu}(p)\left(p^{2}\right)^{\eta_{1}-d / 2} .
$$

As for the conserved vector currents, also for the energy momentum tensor the scaling dimension is fixed by the second of Eq.(12) and it is given by $\eta_{1}=d$. This particular value ensures that $\partial^{\mu} T_{\mu \nu}$ is also a quasi primary (vector) field. We have left to the appendix B the details of the characterization of the vector and tensor two-point functions.

These formulae agree with those in the literature [16], and in particular those in Sec. 8 of Ref. [12] for the gravitational wave spectrum of the CMB.

\subsection{Divergences and anomalous breaking of scale identities}

The expressions obtained so far for the two-point functions in Eq.(15),(18) and (21), allow to discuss very easily the question of the divergences and of the corresponding violations that these induce in the scale identities. We can naturally see this noting that the Gamma function has simple poles for non positive integer arguments, which occur, in our case, when $\eta=d / 2+n$ with $n=0,1,2, \ldots$

Working in dimensional regularization, we can parametrize the divergence through an analytic continuation of the space-time dimension, $d \rightarrow d-2 \epsilon$, and, then, expand the product $\Gamma(d / 2-$ $\eta)\left(p^{2}\right)^{\eta-d / 2}$, which appears in every two-point function, in a Laurent series around $d / 2-\eta=-n$. We obtain

$$
\Gamma(d / 2-\eta)\left(p^{2}\right)^{\eta-d / 2}=\frac{(-1)^{n}}{n !}\left(-\frac{1}{\epsilon}+\psi(n+1)+O(\epsilon)\right)\left(p^{2}\right)^{n+\epsilon},
$$


where $\psi(z)$ is the logarithmic derivative of the Gamma function, and $\epsilon$ takes into account the divergence of the two-point correlator for particular values of the scale dimension $\eta$ and of the space-time dimension $d$.

The singular behavior described in Eq.(22) is responsible for the anomalous violation of scale invariance [17], providing an extra contribution to the differential equation (12) obtained from the conformal symmetry constraints. Indeed, when $\eta=d / 2+n$, employing dimensional regularization, the first of Eq.(12) becomes

$$
\left(p^{2} \frac{\partial}{\partial p^{2}}-n-\epsilon\right) G^{i j}\left(p^{2}\right)=0, \quad \text { with } \quad \eta_{1}=\eta_{2} \equiv \eta
$$

which is the Euler equation for a function $G^{i j}$ which behaves like $\left(p^{2}\right)^{n+\epsilon}$. Due to the appearance of a divergence in $1 / \epsilon$ in the correlation function, Eq.(23) acquires an anomalous finite term in the limit $\epsilon \rightarrow 0$ and we obtain

$$
\left(p^{2} \frac{\partial}{\partial p^{2}}-n\right) G^{i j}\left(p^{2}\right)=G_{\text {sing }}^{i j}\left(p^{2}\right)
$$

where $G_{\text {sing }}^{i j}\left(p^{2}\right)$ corresponds to the singular contribution in the correlation function, which we have decomposed according to

$$
G^{i j}\left(p^{2}\right)=\frac{1}{\epsilon} G_{\text {sing }}^{i j}\left(p^{2}\right)+G_{\text {finite }}^{i j}\left(p^{2}\right) .
$$

As one can see from the r.h.s. of Eq.(24), the coefficient of the divergence, $G_{s i n g}^{i j}\left(p^{2}\right)$, of the two-point function provides the source for its anomalous scaling.

We illustrate the points discussed so far with some examples. Consider, for instance, the scalar correlator in Eq.(15) with scaling dimension $\eta_{1}=\eta_{2} \equiv \eta=d / 2$. Due to the appearance of a pole in the Gamma function, the two-point correlator develops a divergence and becomes

$$
G_{S}\left(p^{2}\right)=-c_{S 12} \frac{\pi^{d / 2}}{\Gamma(d / 2)}\left[\frac{1}{\bar{\epsilon}}+\log p^{2}\right],
$$

where we have defined for convenience

$$
\frac{1}{\bar{\epsilon}}=\frac{1}{\epsilon}+\gamma-\log (4 \pi)
$$

with $\gamma$ being the Euler-Mascheroni constant. It is implicitly understood that the argument of the logarithm in Eq.(26) is made dimensionless, in dimensional regularization, by the insertion of a massive parameter.

As one can easily verify, the scalar two-point function given in Eq.(26) satisfies the anomalous scaling equation (24) with a constant source term

$$
G_{S, s i n g}\left(p^{2}\right)=-c_{S 12} \frac{\pi^{d / 2}}{\Gamma(d / 2)}
$$


determined by the coefficient of the singularity. Note that the anomalous scaling behavior in Eq.(24) is reproduced by the logarithmic contribution in Eq.(26) $)$.

Now we turn to the discussion of a correlation function with two vector currents. As already mentioned, the scaling dimension of the conserved vector operator is fixed at the value $\eta=d-1$. In this case the divergences occur at $d=2 n+2$ with $n=0,1, \ldots$, so that, for $d>2$, the first singularity appears at $d=4$. Therefore the vector two-point function for $d=4$ is

$$
G_{V}^{\alpha \beta}\left(p^{2}\right)=c_{V 12} \frac{\pi^{2}}{8} p^{2}\left[\frac{1}{\bar{\epsilon}}-1+\log p^{2}\right] \pi^{\alpha \beta}(p)
$$

with $\bar{\epsilon}$ defined in Eq.(27). As for the previous case, it is manifest that the two-point function in Eq.(29) satisfies the identity given in Eq.(24), with the logarithm accounting for the source of the anomalous scaling behavior.

Finally, we illustrate the case of the correlation function built with two (symmetric, conserved and traceless) energy momentum tensors with scale dimension $\eta=d$, which is slightly more involved, as we have to pay attention to the fact that $\Pi_{d}^{\alpha \beta \mu \nu}(p)$ itself depends on the spacetime dimension $d$. The singularities are generated when $d=2 n$ with $n=0,1, \ldots$, namely for even values of the space-time dimension. For instance, the two-point function in $d=4$ is found to be given by

$$
G_{T}^{\alpha \beta \mu \nu}(p)=-c_{T 12} \frac{\pi^{2}}{192}\left(p^{2}\right)^{2}\left\{\left[\frac{1}{\bar{\epsilon}}-\frac{3}{2}+\log p^{2}\right] \Pi_{4}^{\alpha \beta \mu \nu}(p)-\frac{2}{9} \pi^{\alpha \beta}(p) \pi^{\mu \nu}(p)\right\} .
$$

As we have already discussed previously, the appearance of the singularity in the correlation function develops an anomalous term in the scale identity. Correspondingly, being the energy momentum tensor related to the dilatation current, $J_{D}^{\mu}=x_{\nu} T^{\mu \nu}$, it acquires an anomalous trace reflecting the violation of the scale symmetry. In this respect, the two-point function in Eq.(30) is characterized by a non vanishing trace

$$
\eta_{\mu \nu} G_{T}^{\alpha \beta \mu \nu}(p)=c_{T 12} \frac{\pi^{2}}{288}\left(p^{2}\right)^{2} \pi^{\alpha \beta}(p)
$$

generated by the last term in Eq.(30) which, on the other hand, arises from the explicit dependence of the $\Pi_{d}^{\alpha \beta \mu \nu}(p)$ tensor on the space-time dimension. The non-zero trace of Eq.(31) is the signature of a conformal or trace anomaly, whose coefficients are known for free fields [18, 19].

\section{Three-point functions for scalar operators}

In this section we turn to the momentum space analysis of conformal invariant three-point functions, by solving the constraints emerging from the invariance under the conformal group. 
We consider scalar quasi primary fields $\mathcal{O}_{i}$ with scale dimensions $\eta_{i}$ and define the three-point function

$$
G_{123}\left(p_{1}, p_{2}\right)=\left\langle\mathcal{O}_{1}\left(p_{1}\right) \mathcal{O}_{2}\left(p_{2}\right) \mathcal{O}_{3}\left(-p_{1}-p_{2}\right)\right\rangle
$$

The three-point correlator is a function of the two independent momenta $p_{1}$ and $p_{2}$, from which one can construct three independent scalar quantities, namely $p_{1}^{2}, p_{2}^{2}$ and $p_{1} \cdot p_{2}$. We trade the last invariant for $p_{3}^{2}$ in order to manifest the symmetry properties of $G_{123}$ under the exchange of any couple of operators.

We observe that scale invariance, the first equation in Eq.(11), implies that $G_{123}$ is a homogeneous function of degree $\alpha=-d+\frac{1}{2}\left(\eta_{1}+\eta_{2}+\eta_{3}\right)$. Therefore it can be written in the form

$$
G_{123}\left(p_{1}^{2}, p_{2}^{2}, p_{3}^{2}\right)=\left(p_{3}^{2}\right)^{-d+\frac{1}{2}\left(\eta_{1}+\eta_{2}+\eta_{3}\right)} \Phi(x, y) \quad \text { with } \quad x=\frac{p_{1}^{2}}{p_{3}^{2}}, \quad y=\frac{p_{2}^{2}}{p_{3}^{2}}
$$

where we have introduced the dimensionless ratios $x$ and $y$, which must not be confused with coordinate points. The dilatation equation only fixes the scaling behavior of the three-point correlator giving no further information on the dimensionless function $\Phi(x, y)$.

The last equation of (11), which describes the invariance under special conformal transformations, is the most predictive one and, as we shall see, completely determines $\Phi(x, y)$ up to a multiplicative constant.

To show this, we start by rewriting Eq.(11) in a more useful form by introducing a change of variables from $\left(p_{1}^{2}, p_{2}^{2}, p_{3}^{2}\right)$ to $\left(x, y, p_{3}^{2}\right)$. The derivatives respect to the momentum components are re-expressed in terms of derivatives of the momentum invariants and their ratios as

$$
\begin{aligned}
\frac{\partial}{\partial p_{1}^{\mu}} & =2\left(p_{1 \mu}+p_{2 \mu}\right) \frac{\partial}{\partial p_{3}^{2}}+\frac{2}{p_{3}^{2}}\left((1-x) p_{1 \mu}-x p_{2 \mu}\right) \frac{\partial}{\partial x}-2\left(p_{1 \mu}+p_{2 \mu}\right) \frac{y}{p_{3}^{2}} \frac{\partial}{\partial y} \\
\frac{\partial}{\partial p_{2}^{\mu}} & =2\left(p_{1 \mu}+p_{2 \mu}\right) \frac{\partial}{\partial p_{3}^{2}}-2\left(p_{1 \mu}+p_{2 \mu}\right) \frac{x}{p_{3}^{2}} \frac{\partial}{\partial x}+\frac{2}{p_{3}^{2}}\left((1-y) p_{2 \mu}-y p_{1 \mu}\right) \frac{\partial}{\partial y}
\end{aligned}
$$

Similar but lengthier formulas hold for second derivatives. Also notice that the derivatives with respect to $p_{3}^{2}$ can be removed using the solution of the dilatation constraint in Eq.(33). Therefore we are left with a differential equation in the two dimensionless variables $x$ and $y$. Due to the vector nature of the special conformal transformations, Eq.(11) can be projected out on the two independent momenta $p_{1}$ and $p_{2}$, obtaining a system of two coupled second order partial differential equations $(\mathrm{PDE})$ for the function $\Phi(x, y)$. After several non trivial 
manipulations, these can be recast in the simple form

$$
\left\{\begin{aligned}
& {\left[x(1-x) \frac{\partial^{2}}{\partial x^{2}}-y^{2} \frac{\partial^{2}}{\partial y^{2}}-2 x y \frac{\partial^{2}}{\partial x \partial y}+[\gamma-(\alpha+\beta+1) x] \frac{\partial}{\partial x}\right.}\left.-(\alpha+\beta+1) y \frac{\partial}{\partial y}-\alpha \beta\right] \Phi(x, y)=0 \\
& {\left[y(1-y) \frac{\partial^{2}}{\partial y^{2}}-x^{2} \frac{\partial^{2}}{\partial x^{2}}-2 x y \frac{\partial^{2}}{\partial x \partial y}+\left[\gamma^{\prime}-(\alpha+\beta+1) y\right] \frac{\partial}{\partial y}\right.} \\
&\left.-(\alpha+\beta+1) x \frac{\partial}{\partial x}-\alpha \beta\right] \Phi(x, y)=0
\end{aligned}\right.
$$

with the parameters $\alpha, \beta, \gamma, \gamma^{\prime}$ defined in terms of the scale dimensions of the three scalar operators as

$$
\begin{array}{ll}
\alpha=\frac{d}{2}-\frac{\eta_{1}+\eta_{2}-\eta_{3}}{2}, & \gamma=\frac{d}{2}-\eta_{1}+1, \\
\beta=d-\frac{\eta_{1}+\eta_{2}+\eta_{3}}{2}, & \gamma^{\prime}=\frac{d}{2}-\eta_{2}+1 .
\end{array}
$$

It is interesting to observe that the system of equations in (35), coming from the invariance under special conformal transformations, is exactly the system of partial differential equations defining the hypergeometric Appell's function of two variables, $F_{4}\left(\alpha, \beta ; \gamma, \gamma^{\prime} ; x, y\right)$, with coefficients given in Eq.(36). The Appell's function $F_{4}$ is defined as the double series (see, e.g., [20, 21, 22] for thorough discussions of the hypergeometric functions and their properties)

$$
F_{4}\left(\alpha, \beta ; \gamma, \gamma^{\prime} ; x, y\right)=\sum_{i=0}^{\infty} \sum_{j=0}^{\infty} \frac{(\alpha)_{i+j}(\beta)_{i+j}}{(\gamma)_{i}\left(\gamma^{\prime}\right)_{j}} \frac{x^{i}}{i !} \frac{y^{j}}{j !}
$$

where $(\alpha)_{i}=\Gamma(\alpha+i) / \Gamma(\alpha)$ is the Pochhammer symbol.

It is known that the system of partial differential equations (35), besides the hypergeometric function introduced in Eq.(37), has three other independent solutions given by

$$
\begin{aligned}
& S_{2}\left(\alpha, \beta ; \gamma, \gamma^{\prime} ; x, y\right)=x^{1-\gamma} F_{4}\left(\alpha-\gamma+1, \beta-\gamma+1 ; 2-\gamma, \gamma^{\prime} ; x, y\right) \\
& S_{3}\left(\alpha, \beta ; \gamma, \gamma^{\prime} ; x, y\right)=y^{1-\gamma^{\prime}} F_{4}\left(\alpha-\gamma^{\prime}+1, \beta-\gamma^{\prime}+1 ; \gamma, 2-\gamma^{\prime} ; x, y\right) \\
& S_{4}\left(\alpha, \beta ; \gamma, \gamma^{\prime} ; x, y\right)=x^{1-\gamma} y^{1-\gamma^{\prime}} F_{4}\left(\alpha-\gamma-\gamma^{\prime}+2, \beta-\gamma-\gamma^{\prime}+2 ; 2-\gamma, 2-\gamma^{\prime} ; x, y\right)
\end{aligned}
$$

Therefore the function $\Phi(x, y)$, solution of (35), is a linear combination of the four independent hypergeometric functions, i.e.

$$
\begin{aligned}
G_{123}\left(p_{1}^{2}, p_{2}^{2}, p_{3}^{2}\right) & =\left(p_{3}^{2}\right)^{-d+\frac{1}{2}\left(\eta_{1}+\eta_{2}+\eta_{3}\right)} \Phi(x, y) \\
& =\left(p_{3}^{2}\right)^{-d+\frac{1}{2}\left(\eta_{1}+\eta_{2}+\eta_{3}\right)} \sum_{i=1}^{4} c_{i}\left(\eta_{1}, \eta_{2}, \eta_{3}\right) S_{i}\left(\alpha, \beta ; \gamma, \gamma^{\prime} ; x, y\right)
\end{aligned}
$$


where we have denoted with $S_{1}$ the Appell's function $F_{4}$ given in Eq.(37), while the parameters $\alpha, \beta, \gamma, \gamma^{\prime}$ are defined in Eq.(36). The $c_{i}\left(\eta_{1}, \eta_{2}, \eta_{3}\right)$ appearing in the linear combination, are the arbitrary coefficients which may depend on the scale dimensions $\eta_{i}$ of the quasi primary fields and on the space-time dimension $d$.

The coefficients $c_{i}\left(\eta_{1}, \eta_{2}, \eta_{3}\right)$ can be determined, up to an overall multiplicative constant, by exploiting the symmetry of the correlation function under the interchange of two of the three scalar operators present in the correlator, which consists of the simultaneous exchange of momenta and scale dimensions $\left(p_{i}^{2}, \eta_{i}\right) \leftrightarrow\left(p_{j}^{2}, \eta_{j}\right)$.

Consider, for instance, the invariance of the three-point function under the exchange $\mathcal{O}_{2}\left(p_{2}\right) \leftrightarrow$ $\mathcal{O}_{3}\left(-p_{1}-p_{2}\right)$, which is achieved by $\left(p_{2}^{2}, \eta_{2}\right) \leftrightarrow\left(p_{3}^{2}, \eta_{3}\right)$. Then Eq.(39) becomes

$$
G_{132}\left(p_{1}^{2}, p_{3}^{2}, p_{2}^{2}\right)=\left(p_{2}^{2}\right)^{-d+\frac{1}{2}\left(\eta_{1}+\eta_{2}+\eta_{3}\right)} \sum_{i=1}^{4} c_{i}\left(\eta_{1}, \eta_{3}, \eta_{2}\right) S_{i}\left(\tilde{\alpha}, \tilde{\beta} ; \tilde{\gamma}, \tilde{\gamma}^{\prime} ; \frac{x}{y}, \frac{1}{y}\right)
$$

where

$$
\begin{array}{ll}
\tilde{\alpha}=\alpha\left(\eta_{2} \leftrightarrow \eta_{3}\right)=\frac{d}{2}-\frac{\eta_{1}+\eta_{3}-\eta_{2}}{2}, & \tilde{\gamma}=\gamma\left(\eta_{2} \leftrightarrow \eta_{3}\right)=\frac{d}{2}-\eta_{1}+1=\gamma, \\
\tilde{\beta}=\beta\left(\eta_{2} \leftrightarrow \eta_{3}\right)=d-\frac{\eta_{1}+\eta_{2}+\eta_{3}}{2}=\beta, & \tilde{\gamma}^{\prime}=\gamma^{\prime}\left(\eta_{2} \leftrightarrow \eta_{3}\right)=\frac{d}{2}-\eta_{3}+1 .
\end{array}
$$

Note that the hypergeometric functions are now evaluated in $x / y$ and $1 / y$. To reintroduce the dependence from $x$ and $y$, in order to exploit more easily the symmetry relation

$$
G_{123}\left(p_{1}^{2}, p_{2}^{2}, p_{3}^{2}\right)=G_{132}\left(p_{1}^{2}, p_{3}^{2}, p_{2}^{2}\right)
$$

we make use of the transformation property of $F_{4}[20]$

$$
\begin{aligned}
F_{4}\left(\alpha, \beta ; \gamma, \gamma^{\prime} ; x, y\right) & =\frac{\Gamma\left(\gamma^{\prime}\right) \Gamma(\beta-\alpha)}{\Gamma\left(\gamma^{\prime}-\alpha\right) \Gamma(\beta)}(-y)^{-\alpha} F_{4}\left(\alpha, \alpha-\gamma^{\prime}+1 ; \gamma, \alpha-\beta+1 ; \frac{x}{y}, \frac{1}{y}\right) \\
& +\frac{\Gamma\left(\gamma^{\prime}\right) \Gamma(\alpha-\beta)}{\Gamma\left(\gamma^{\prime}-\beta\right) \Gamma(\alpha)}(-y)^{-\beta} F_{4}\left(\beta-\gamma^{\prime}+1, \beta ; \gamma, \beta-\alpha+1 ; \frac{x}{y}, \frac{1}{y}\right)
\end{aligned}
$$

After some algebraic manipulations, and repeating the procedure described so far for the other operator interchanges, the ratios between the coefficients $c_{i}$ take the simplified form

$$
\begin{aligned}
& \frac{c_{1}\left(\eta_{1}, \eta_{2}, \eta_{3}\right)}{c_{3}\left(\eta_{1}, \eta_{2}, \eta_{3}\right)}=\frac{\Gamma\left(\eta_{2}-\frac{d}{2}\right) \Gamma\left(d-\frac{\eta_{1}}{2}-\frac{\eta_{2}}{2}-\frac{\eta_{3}}{2}\right) \Gamma\left(\frac{d}{2}-\frac{\eta_{1}}{2}-\frac{\eta_{2}}{2}+\frac{\eta_{3}}{2}\right)}{\Gamma\left(\frac{d}{2}-\eta_{2}\right) \Gamma\left(-\frac{\eta_{1}}{2}+\frac{\eta_{2}}{2}+\frac{\eta_{3}}{2}\right) \Gamma\left(\frac{d}{2}-\frac{\eta_{1}}{2}+\frac{\eta_{2}}{2}-\frac{\eta_{3}}{2}\right)}, \\
& \frac{c_{2}\left(\eta_{1}, \eta_{2}, \eta_{3}\right)}{c_{4}\left(\eta_{1}, \eta_{2}, \eta_{3}\right)}=\frac{\Gamma\left(\eta_{2}-\frac{d}{2}\right) \Gamma\left(\frac{\eta_{1}}{2}-\frac{\eta_{2}}{2}+\frac{\eta_{3}}{2}\right) \Gamma\left(\frac{d}{2}+\frac{\eta_{1}}{2}-\frac{\eta_{2}}{2}-\frac{\eta_{3}}{2}\right)}{\Gamma\left(\frac{d}{2}-\eta_{2}\right) \Gamma\left(\frac{\eta_{1}}{2}+\frac{\eta_{2}}{2}-\frac{\eta_{3}}{2}\right) \Gamma\left(-\frac{d}{2}+\frac{\eta_{1}}{2}+\frac{\eta_{2}}{2}+\frac{\eta_{3}}{2}\right)}, \\
& \frac{c_{1}\left(\eta_{1}, \eta_{2}, \eta_{3}\right)}{c_{4}\left(\eta_{1}, \eta_{2}, \eta_{3}\right)}=\frac{\Gamma\left(\eta_{1}-\frac{d}{2}\right) \Gamma\left(\eta_{2}-\frac{d}{2}\right) \Gamma\left(d-\frac{\eta_{1}}{2}-\frac{\eta_{2}}{2}-\frac{\eta_{3}}{2}\right) \Gamma\left(\frac{d}{2}-\frac{\eta_{1}}{2}-\frac{\eta_{2}}{2}+\frac{\eta_{3}}{2}\right)}{\Gamma\left(\frac{d}{2}-\eta_{1}\right) \Gamma\left(\frac{d}{2}-\eta_{2}\right) \Gamma\left(\frac{\eta_{1}}{2}+\frac{\eta_{2}}{2}-\frac{\eta_{3}}{2}\right) \Gamma\left(-\frac{d}{2}+\frac{\eta_{1}}{2}+\frac{\eta_{2}}{2}+\frac{\eta_{3}}{2}\right)},
\end{aligned}
$$


and define $G_{123}\left(p_{1}^{2}, p_{2}^{2}, p_{3}^{2}\right)$ up to a multiplicative arbitrary constant $c_{123} \equiv c_{123}\left(\eta_{1}, \eta_{2}, \eta_{3}\right)$. This depends on the space-time dimension $d$, on the scale dimensions $\eta_{i}$ of the quasi primary fields and on their normalization.

The conformal invariant correlation function of three scalar quasi primary fields with arbitrary scale dimensions is then given by

$$
\begin{aligned}
G_{123}\left(p_{1}^{2},\right. & \left.p_{2}^{2}, p_{3}^{2}\right)=\frac{c_{123} \pi^{d} 4^{d-\frac{1}{2}\left(\eta_{1}+\eta_{2}+\eta_{3}\right)}\left(p_{3}^{2}\right)^{-d+\frac{1}{2}\left(\eta_{1}+\eta_{2}+\eta_{3}\right)}}{\Gamma\left(\frac{\eta_{1}}{2}+\frac{\eta_{2}}{2}-\frac{\eta_{3}}{2}\right) \Gamma\left(\frac{\eta_{1}}{2}-\frac{\eta_{2}}{2}+\frac{\eta_{3}}{2}\right) \Gamma\left(-\frac{\eta_{1}}{2}+\frac{\eta_{2}}{2}+\frac{\eta_{3}}{2}\right) \Gamma\left(-\frac{d}{2}+\frac{\eta_{1}}{2}+\frac{\eta_{2}}{2}+\frac{\eta_{3}}{2}\right)}\{ \\
& \Gamma\left(\eta_{1}-\frac{d}{2}\right) \Gamma\left(\eta_{2}-\frac{d}{2}\right) \Gamma\left(d-\frac{\eta_{1}}{2}-\frac{\eta_{2}}{2}-\frac{\eta_{3}}{2}\right) \Gamma\left(\frac{d}{2}-\frac{\eta_{1}}{2}-\frac{\eta_{2}}{2}+\frac{\eta_{3}}{2}\right) \\
& \times F_{4}\left(\frac{d}{2}-\frac{\eta_{1}+\eta_{2}-\eta_{3}}{2}, d-\frac{\eta_{1}+\eta_{2}+\eta_{3}}{2} ; \frac{d}{2}-\eta_{1}+1, \frac{d}{2}-\eta_{2}+1 ; x, y\right) \\
& +\Gamma\left(\frac{d}{2}-\eta_{1}\right) \Gamma\left(\eta_{2}-\frac{d}{2}\right) \Gamma\left(\frac{\eta_{1}}{2}-\frac{\eta_{2}}{2}+\frac{\eta_{3}}{2}\right) \Gamma\left(\frac{d}{2}+\frac{\eta_{1}}{2}-\frac{\eta_{2}}{2}-\frac{\eta_{3}}{2}\right) \\
& \times x^{\eta_{1}-\frac{d}{2}} F_{4}\left(\frac{d}{2}-\frac{\eta_{2}+\eta_{3}-\eta_{1}}{2}, \frac{\eta_{1}+\eta_{3}-\eta_{2}}{2} ;-\frac{d}{2}+\eta_{1}+1, \frac{d}{2}-\eta_{2}+1 ; x, y\right) \\
& +\Gamma\left(\eta_{1}-\frac{d}{2}\right) \Gamma\left(\frac{d}{2}-\eta_{2}\right) \Gamma\left(-\frac{\eta_{1}}{2}+\frac{\eta_{2}}{2}+\frac{\eta_{3}}{2}\right) \Gamma\left(\frac{d}{2}-\frac{\eta_{1}}{2}+\frac{\eta_{2}}{2}-\frac{\eta_{3}}{2}\right) \\
& \times y^{\eta_{2}-\frac{d}{2}} F_{4}\left(\frac{d}{2}-\frac{\eta_{1}+\eta_{3}-\eta_{2}}{2}, \frac{\eta_{2}+\eta_{3}-\eta_{1}}{2} ; \frac{d}{2}-\eta_{1}+1,-\frac{d}{2}+\eta_{2}+1 ; x, y\right) \\
& +\Gamma\left(\frac{d}{2}-\eta_{1}\right) \Gamma\left(\frac{d}{2}-\eta_{2}\right) \Gamma\left(\frac{\eta_{1}}{2}+\frac{\eta_{2}}{2}-\frac{\eta_{3}}{2}\right) \Gamma\left(-\frac{d}{2}+\frac{\eta_{1}}{2}+\frac{\eta_{2}}{2}+\frac{\eta_{3}}{2}\right) \\
& \left.\times x^{\eta_{1}-\frac{d}{2}} y^{\eta_{2}-\frac{d}{2}} F_{4}\left(-\frac{d}{2}+\frac{\eta_{1}+\eta_{2}+\eta_{3}}{2}, \frac{\eta_{1}+\eta_{2}-\eta_{3}}{2} ;-\frac{d}{2}+\eta_{1}+1,-\frac{d}{2}+\eta_{2}+1 ; x, y\right)\right\} .
\end{aligned}
$$

The convenient normalization employed in Eq.(45) for the three-point function reproduces, through the operator product expansion, as we are going to show next, the normalization of the two-point functions which we have chosen in Eq.(15).

As we shall identify the three-point correlator discussed in this section with specific Feynman amplitudes, this will fix the arbitrary constant $c_{123}$ using some information coming from the same operator product expansion analysis. This topic will be presented in section 5. Indeed, the solution of the momentum space version of the conformal constraints provides an alternative computational tool for correlation functions with conformal symmetry.

It is worth to emphasize the connection between the invariance under special conformal transformations and appearance of the Appell's functions. Indeed we have shown how the constraints provided by the conformal group translate, in momentum space, in the well-known system of partial differential equations defining the hypergeometric series $F_{4}$. We have analyzed this connection in the case of a conformally invariant three-point function built with scalar operators in some detail. A similar correspondence should also hold for more complicated 
vector and tensor correlators.

\subsection{The Operator Product Expansion analysis}

In this section we show the consistency of our result with the operator product expansion (OPE) in conformal field theories in which the structure of the Wilson's coefficients is entirely fixed by the scaling dimensions of the two operators.

Considering, for instance, the coincidence limit in the scalar case, one has

$$
\mathcal{O}_{i}\left(x_{1}\right) \mathcal{O}_{j}\left(x_{2}\right) \sim \sum_{k} \frac{c_{i j k}}{\left(x_{12}^{2}\right)^{\frac{1}{2}\left(\eta_{i}+\eta_{j}-\eta_{k}\right)}} \mathcal{O}_{k}\left(x_{2}\right) \quad \text { for } \quad x_{1} \rightarrow x_{2},
$$

where $x_{12}=x_{1}-x_{2}$. It is worth noting that the coefficients $c_{i j k}$ are the same structure constants appearing in the three-point functions.

For the correlation function of three scalar operators the OPE implies the singular behavior

$$
\left\langle\mathcal{O}_{1}\left(x_{1}\right) \mathcal{O}_{2}\left(x_{2}\right) \mathcal{O}_{3}\left(x_{3}\right)\right\rangle \stackrel{x_{3} \rightarrow^{x_{2}}}{\sim} \frac{c_{123}}{\left(x_{23}^{2}\right)^{\frac{1}{2}\left(\eta_{2}+\eta_{3}-\eta_{1}\right)}}\left\langle\mathcal{O}_{1}\left(x_{1}\right) \mathcal{O}_{2}\left(x_{2}\right)\right\rangle
$$

with analogous formulae for the other coincidence limits. For the sake of simplicity, we choose a diagonal basis of quasi primary operators normalized as

$$
\left\langle\mathcal{O}_{i}\left(x_{1}\right) \mathcal{O}_{j}\left(x_{2}\right)\right\rangle=\frac{\delta_{i j}}{\left(x_{12}^{2}\right)^{\eta_{i}}} .
$$

The momentum space version of the OPE in Eq.(47) reads

$$
\begin{aligned}
\left\langle\mathcal{O}_{1}\left(p_{1}\right) \mathcal{O}_{2}\left(p_{2}\right) \mathcal{O}_{3}\left(-p_{1}-p_{2}\right)\right\rangle & \\
& \sim \frac{\pi^{d / 2}}{4^{\frac{1}{2}\left(\eta_{2}+\eta_{3}-\eta_{1}\right)-\frac{d}{2}}} \frac{\Gamma\left(\frac{d}{2}-\frac{\eta_{2}+\eta_{3}-\eta_{1}}{2}\right)}{\Gamma\left(\frac{\eta_{2}+\eta_{3}-\eta_{1}}{2}\right)} \frac{c_{123}}{\left(p_{3}^{2}\right)^{\frac{d}{2}-\frac{1}{2}\left(\eta_{2}+\eta_{3}-\eta_{1}\right)}}\left\langle\mathcal{O}_{1}\left(p_{1}\right) \mathcal{O}_{2}\left(-p_{1}\right)\right\rangle,
\end{aligned}
$$

where the scalar two-point function is normalized as in Eq.(15) with $c_{S 12}=1$. In the previous equation the symbol $\sim$ stands for the momentum space counterpart of the short distance limit $x_{3} \rightarrow x_{2}$ which is achieved by the $p_{3}^{2}, p_{2}^{2} \rightarrow \infty$ limit with $p_{2}^{2} / p_{3}^{2} \rightarrow 1$.

The result for the scalar three-point function given in Eq.(45) is indeed in agreement, as expected, with the OPE analysis. This can be shown from Eq.(45) by a suitable expansion of the corresponding Appell's functions. In particular, in order to reproduce the momentum space singular behavior of Eq.(49), we need the hypergeometric leading expansion in the limit $x=p_{1}^{2} / p_{3}^{2} \rightarrow 0$ and $y=p_{2}^{2} / p_{3}^{2} \rightarrow 1$, which reads as [20]

$$
F_{4}\left(\alpha, \beta ; \gamma, \gamma^{\prime} ; x, y\right) \sim \frac{\Gamma\left(\gamma^{\prime}\right) \Gamma\left(\gamma^{\prime}-\alpha-\beta\right)}{\Gamma\left(\gamma^{\prime}-\alpha\right) \Gamma\left(\gamma^{\prime}-\beta\right)} \quad \text { for } \quad x \rightarrow 0, y \rightarrow 1
$$


In the previous equation we have retained only the terms with the correct power-law scaling in the $p_{3}^{2}$ variable, as dictated by the OPE analysis. In this case these contributions come from the terms of Eq.(45) which are proportional to the $S_{2}$ and $S_{4}$ solutions defined in Eq.(38). Analogously, in the limit $p_{3}^{2}, p_{1}^{2} \rightarrow \infty$, with $p_{1}^{2} / p_{3}^{2} \rightarrow 1$, which is described in coordinate space by $x_{3} \rightarrow x_{1}$, the leading behavior is extracted from $S_{3}$ and $S_{4}$.

The remaining coincidence limit $x_{1} \rightarrow x_{2}$, corresponding to $p_{1}^{2}, p_{2}^{2} \rightarrow \infty$ with $p_{1}^{2} / p_{2}^{2} \rightarrow 1$, is more subtle due to the apparent asymmetry in the momentum invariants $p_{1}^{2}, p_{2}^{2}, p_{3}^{2}$ of the three-point scalar correlator, as given in Eq.(45). In this case both $x$ and $y$ grow to infinity while their ratio $x / y \rightarrow 1$. Therefore it is necessary to apply the transformation defined in Eq.(43) to each hypergeometric function appearing in Eq.(45). This can be viewed as an analytic continuation outside the domain of convergence $|\sqrt{x}|+|\sqrt{y}|<1$, where the Appell's function is strictly defined as a double series. The hypergeometric functions are then expanded according to

$$
\begin{aligned}
F_{4}\left(\alpha, \beta ; \gamma, \gamma^{\prime} ; x, y\right) & \sim(-y)^{-\alpha} \frac{\Gamma(\gamma) \Gamma\left(\gamma^{\prime}\right) \Gamma(\beta-\alpha) \Gamma\left(\gamma+\gamma^{\prime}-2 \alpha-1\right)}{\Gamma(\beta) \Gamma(\gamma-\alpha) \Gamma\left(\gamma^{\prime}-\alpha\right) \Gamma\left(\gamma+\gamma^{\prime}-\alpha-1\right)} \\
& +\quad(-y)^{-\beta} \frac{\Gamma(\gamma) \Gamma\left(\gamma^{\prime}\right) \Gamma(\alpha-\beta) \Gamma\left(\gamma+\gamma^{\prime}-2 \beta-1\right)}{\Gamma(\alpha) \Gamma\left(\gamma^{\prime}-\beta\right) \Gamma(\gamma-\beta) \Gamma\left(\gamma+\gamma^{\prime}-\beta-1\right)} \\
& \text { for } \quad x, y \rightarrow \infty, \frac{x}{y} \rightarrow 1 .
\end{aligned}
$$

This completes the analysis of the OPE on the three-point scalar function in the three different coincidence limits.

\section{Feynman integral representation of the momentum space solution}

We have seen in the previous sections that we can fix the explicit structure of the generic three-point scalar correlator in momentum space by solving the conformal constraints, which are mapped to a system of two hypergeometric differential equations of two variables. These variables take the form of two ratios of the external momenta. In particular we find that in any $d$ dimensional conformal field theory the solution of this system of PDE's is characterized by a single integration constant which depends on the specific conformal realization, as expected.

In this section we want to point out the relationship between the scalar three-point functions studied so far and a certain class of Feynman master integrals. These can be obtained by a Fourier transformation of the corresponding solution of the conformal constraints in coordinate space, which is well known to be

$$
\left\langle\mathcal{O}_{1}\left(x_{1}\right) \mathcal{O}_{2}\left(x_{2}\right) \mathcal{O}_{3}\left(x_{3}\right)\right\rangle=\frac{c_{123}}{\left(x_{12}^{2}\right)^{\frac{1}{2}\left(\eta_{1}+\eta_{2}-\eta_{3}\right)}\left(x_{23}^{2}\right)^{\frac{1}{2}\left(\eta_{2}+\eta_{3}-\eta_{1}\right)}\left(x_{31}^{2}\right)^{\frac{1}{2}\left(\eta_{3}+\eta_{1}-\eta_{2}\right)}} .
$$


Transforming to momentum space, we find an integral representation, which necessarily has to coincide, up to an unconstrained overall constant, with the explicit solution found in the previous section, and reads as

$$
J\left(\nu_{1}, \nu_{2}, \nu_{3}\right)=\int \frac{d^{d} l}{(2 \pi)^{d}} \frac{1}{\left(l^{2}\right)^{\nu_{3}}\left(\left(l+p_{1}\right)^{2}\right)^{\nu_{2}}\left(\left(l-p_{2}\right)^{2}\right)^{\nu_{1}}},
$$

with external momenta $p_{1}, p_{2}$ and $p_{3}$ constrained by momentum conservation $p_{1}+p_{2}+p_{3}=0$ and the scale dimensions $\eta_{i}$ related to the indices $\nu_{i}$ as

$$
\eta_{1}=d-\nu_{2}-\nu_{3}, \quad \eta_{2}=d-\nu_{1}-\nu_{3}, \quad \eta_{3}=d-\nu_{1}-\nu_{2}
$$

This expression describes a family of master integrals which has been studied in [23, 24], whose explicit relation with Eq. (152) is given by

$$
\begin{aligned}
& \int \frac{d^{d} p_{1}}{(2 \pi)^{d}} \frac{d^{d} p_{2}}{(2 \pi)^{d}} \frac{d^{d} p_{3}}{(2 \pi)^{d}}(2 \pi)^{d} \delta^{(d)}\left(p_{1}+p_{2}+p_{3}\right) J\left(\nu_{1}, \nu_{2}, \nu_{3}\right) e^{-i p_{1} \cdot x_{1}-i p_{2} \cdot x_{2}-i p_{3} \cdot x_{3}} \\
& =\frac{1}{4^{\nu_{1}+\nu_{2}+\nu_{3}} \pi^{3 d / 2}} \frac{\Gamma\left(d / 2-\nu_{1}\right) \Gamma\left(d / 2-\nu_{2}\right) \Gamma\left(d / 2-\nu_{3}\right)}{\Gamma\left(\nu_{1}\right) \Gamma\left(\nu_{2}\right) \Gamma\left(\nu_{3}\right)} \frac{1}{\left(x_{12}^{2}\right)^{d / 2-\nu_{3}}\left(x_{23}^{2}\right)^{d / 2-\nu_{1}}\left(x_{31}^{2}\right)^{d / 2-\nu_{2}}},
\end{aligned}
$$

The integral in Eq.(53) satisfies the system of PDE's (35). Therefore, it can be expressed in terms of the general solution given in Eq.(45) which involves a linear combination of four Appell's functions, with the relative coefficients fixed by the symmetry conditions on the dependence from the external momenta. Then Eq.(45) identifies $J\left(\nu_{1}, \nu_{2}, \nu_{3}\right)$ except for an overall constant $c_{123}$ which we are now going to determine. This task can be accomplished, for instance, by exploiting some boundary conditions.

As for the OPE analysis discussed in the previous section, we may consider the large momentum limit in which the three-point integral collapses into a two-point function topology. Taking, for instance, the $p_{2}^{2}, p_{3}^{2} \rightarrow \infty$ limit with $p_{2}^{2} / p_{3}^{2} \rightarrow 1$ we have

$$
J\left(\nu_{1}, \nu_{2}, \nu_{3}\right) \sim \frac{1}{\left(p_{2}^{2}\right)^{\nu_{1}}} \int \frac{d^{d} l}{(2 \pi)^{d}} \frac{1}{\left(l^{2}\right)^{\nu_{3}}\left(\left(l+p_{1}\right)^{2}\right)^{\nu_{2}}}=\frac{1}{\left(p_{2}^{2}\right)^{\nu_{1}}} \frac{i^{1-d}}{(4 \pi)^{d / 2}} G\left(\nu_{2}, \nu_{3}\right)\left(p_{1}^{2}\right)^{d / 2-\nu_{2}-\nu_{3}},
$$

where

$$
G\left(\nu, \nu^{\prime}\right)=\frac{\Gamma(d / 2-\nu) \Gamma\left(d / 2-\nu^{\prime}\right) \Gamma\left(\nu+\nu^{\prime}-d / 2\right)}{\Gamma(\nu) \Gamma\left(\nu^{\prime}\right) \Gamma\left(d-\nu-\nu^{\prime}\right)}
$$

Eq.(56) must be compared with the same limit taken on the explicit solution in Eq.(45), where the scale dimensions $\eta_{i}$ are replaced by $\nu_{i}$ through Eq.(54). This completely determines the 
multiplicative constant $c_{123}$ and the correct normalization of the three-point master integral, which is obtained by choosing

$$
c_{123}=\frac{i^{1-d}}{4^{\nu_{1}+\nu_{2}+\nu_{3}} \pi^{3 d / 2}} \frac{\Gamma\left(d / 2-\nu_{1}\right) \Gamma\left(d / 2-\nu_{2}\right) \Gamma\left(d / 2-\nu_{3}\right)}{\Gamma\left(\nu_{1}\right) \Gamma\left(\nu_{2}\right) \Gamma\left(\nu_{3}\right)} .
$$

Therefore the scalar master integral is given by

$$
J\left(\nu_{1}, \nu_{2}, \nu_{3}\right)=G_{123}\left(p_{1}^{2}, p_{2}^{2}, p_{3}^{2}\right)
$$

with scaling dimensions defined in Eq.(54) and the coefficient $c_{123}$ in Eq.(58). Notice that this method allows us to bypass completely the Mellin-Barnes techniques which has been used previously in the analysis of the same integral.

\subsection{Recurrence relations from conformal invariance}

Having established the conformal invariance of the generalized three-point master integral $J\left(\nu_{1}, \nu_{2}, \nu_{3}\right)$, we can study the implications of the conformal constraints on the integral representation of Eq.(53). These are automatically satisfied by the explicit solution given in Eq.(45), but once that they are applied on $J\left(\nu_{1}, \nu_{2}, \nu_{3}\right)$, generate recursion relations among the indices of this family of integrals. Specifically, they relate integrals with $\nu_{1}+\nu_{2}+\nu_{3}=\kappa$ to those with $\nu_{1}+\nu_{2}+\nu_{3}=\kappa+1$ and $\nu_{1}+\nu_{2}+\nu_{3}=\kappa+2$. For instance, differentiating Eq. (53) under the integration sign according to the first of Eq.(11), which is the condition of scale invariance, we easily obtain the recursion relation

$$
\begin{gathered}
\nu_{2} p_{1}^{2} J\left(\nu_{1}, \nu_{2}+1, \nu_{3}\right)+\nu_{1} p_{2}^{2} J\left(\nu_{1}+1, \nu_{2}, \nu_{3}\right)=\left(\nu_{1}+\nu_{2}+2 \nu_{3}-d\right) J\left(\nu_{1}, \nu_{2}, \nu_{3}\right) \\
+\nu_{2} J\left(\nu_{1}, \nu_{2}+1, \nu_{3}-1\right)+\nu_{1} J\left(\nu_{1}+1, \nu_{2}, \nu_{3}-1\right),
\end{gathered}
$$

together with the corresponding symmetric relations obtained interchanging $\left(p_{1}^{2}, \nu_{1}\right) \leftrightarrow\left(p_{3}^{2}, \nu_{3}\right)$ or $\left(p_{2}^{2}, \nu_{2}\right) \leftrightarrow\left(p_{3}^{2}, \nu_{3}\right)$. These equations link scalar integrals on two contiguous planes, as mentioned above. The recurrence relations obtained from scale invariance exactly correspond to those presented in [24] and following from the usual integration-by-parts technique, which in this case is derived from the divergence theorem in dimensional regularization

$$
\int \frac{d^{d} l}{(2 \pi)^{d}} \frac{\partial}{\partial l_{\mu}}\left\{\frac{l_{\mu}}{\left(l^{2}\right)^{\nu_{3}}\left(\left(l+p_{1}\right)^{2}\right)^{\nu_{2}}\left(\left(l-p_{2}\right)^{2}\right)^{\nu_{1}}}\right\}=0 .
$$

We can easily show the equivalence between Eq.(61) and the first of Eq.(11) which is the constraint of scale invariance. In fact, the scale transformation acts on $J\left(\nu_{1}, \nu_{2}, \nu_{3}\right)$ in the form

$$
\left[d-2\left(\nu_{1}+\nu_{2}+\nu_{3}\right)-p_{1} \cdot \frac{\partial}{\partial p_{1}}-p_{2} \cdot \frac{\partial}{\partial p_{2}}\right] \int d^{d} l \frac{1}{\left(l^{2}\right)^{\nu_{3}}\left(\left(l+p_{1}\right)^{2}\right)^{\nu_{2}}\left(\left(l-p_{2}\right)^{2}\right)^{\nu_{1}}}=0 .
$$


Now we just invoke Euler's theorem on homogeneous functions on the integrand, which is of degree $-2\left(\nu_{1}+\nu_{2}+\nu_{3}\right)$ in the momenta $p_{1}, p_{2}$ and $l$ and obtain the relation

$$
\begin{aligned}
{\left[p_{1} \cdot \frac{\partial}{\partial p_{1}}+p_{2} \cdot \frac{\partial}{\partial p_{2}}+l \cdot \frac{\partial}{\partial l}\right] \frac{1}{\left(l^{2}\right)^{\nu_{3}}\left(\left(l+p_{1}\right)^{2}\right)^{\nu_{2}}} } & \left(\left(l-p_{2}\right)^{2}\right)^{\nu_{1}} \\
& =\frac{-2\left(\nu_{1}+\nu_{2}+\nu_{3}\right)}{\left(l^{2}\right)^{\nu_{3}}\left(\left(l+p_{1}\right)^{2}\right)^{\nu_{2}}\left(\left(l-p_{2}\right)^{2}\right)^{\nu_{1}}} .
\end{aligned}
$$

At this point, if we combine Eqs.(62) and (63) and rewrite $d$ as $\frac{\partial}{\partial l} \cdot l$, we easily obtain the equivalence with Eq.(61).

Other recursive relations can be found requiring Eq.(53) to satisfy the constraint of special conformal invariance which, from the second equation in Eq.(11), takes the form

$$
\left\{p_{1 \mu} \frac{\partial^{2}}{\partial p_{1} \cdot \partial p_{1}}-2 p_{1 \nu} \frac{\partial^{2}}{\partial p_{1}^{\mu} \partial p_{1 \nu}}-2\left(\nu_{2}+\nu_{3}\right) \frac{\partial}{\partial p_{1}^{\mu}}+(1 \leftrightarrow 2)\right\} J\left(\nu_{1}, \nu_{2}, \nu_{3}\right)=0 .
$$

This is a vector condition which involves some tensor integrals of the same $J\left(\nu_{1}, \nu_{2}, \nu_{3}\right)$ family. Differentiating the integral $J\left(\nu_{1}, \nu_{2}, \nu_{3}\right)$ as in Eq.(64) and performing some standard manipulations one arrives at the implicit formula

$$
\begin{aligned}
& \nu_{2} p_{1 \mu}\left[\left(1+\nu_{2}+\nu_{3}-d / 2\right) J\left(\nu_{1}, \nu_{2}+1, \nu_{3}\right)+\left(\nu_{2}+1\right)\left(J\left(\nu_{1}, \nu_{2}+2, \nu_{3}-1\right)-p_{1}^{2} J\left(\nu_{1}, \nu_{2}+2, \nu_{3}\right)\right)\right] \\
& +\nu_{1} p_{2 \mu}\left[\left(1+\nu_{1}+\nu_{3}-d / 2\right) J\left(\nu_{1}+1, \nu_{2}, \nu_{3}\right)+\left(\nu_{1}+1\right)\left(J\left(\nu_{1}+2, \nu_{2}, \nu_{3}-1\right)-p_{2}^{2} J\left(\nu_{1}+2, \nu_{2}, \nu_{3}\right)\right)\right] \\
& +\nu_{2}\left[\left(\nu_{3}-1\right) J_{\mu}\left(\nu_{1}, \nu_{2}+1, \nu_{3}\right)+\left(\nu_{2}+1\right)\left(J_{\mu}\left(\nu_{1}, \nu_{2}+2, \nu_{3}-1\right)-p_{1}^{2} J_{\mu}\left(\nu_{1}, \nu_{2}+2, \nu_{3}\right)\right)\right] \\
& -\nu_{1}\left[\left(\nu_{3}-1\right) J_{\mu}\left(\nu_{1}+1, \nu_{2}, \nu_{3}\right)+\left(\nu_{1}+1\right)\left(J_{\mu}\left(\nu_{1}+2, \nu_{2}, \nu_{3}-1\right)-p_{2}^{2} J_{\mu}\left(\nu_{1}+2, \nu_{2}, \nu_{3}\right)\right)\right]=0,
\end{aligned}
$$

where the rank-1 tensor integral is defined as

$$
J_{\mu}\left(\nu_{1}, \nu_{2}, \nu_{3}\right)=\int \frac{d^{d} l}{(2 \pi)^{d}} \frac{l_{\mu}}{\left(l^{2}\right)^{\nu_{3}}\left(\left(l+p_{1}\right)^{2}\right)^{\nu_{2}}\left(\left(l-p_{2}\right)^{2}\right)^{\nu_{1}}}=C_{1}\left(\nu_{1}, \nu_{2}, \nu_{3}\right) p_{1 \mu}-C_{2}\left(\nu_{1}, \nu_{2}, \nu_{3}\right) p_{2 \mu},
$$

with the coefficients given by

$$
\begin{aligned}
& C_{1}\left(\nu_{1}, \nu_{2}, \nu_{3}\right)=\frac{1}{\left(p_{3}^{2}-p_{1}^{2}-p_{2}^{2}\right)^{2}-4 p_{1}^{2} p_{2}^{2}}\left\{\left(p_{1}^{2}+p_{2}^{2}-p_{3}^{2}\right) J\left(\nu_{1}-1, \nu_{2}, \nu_{3}\right)\right. \\
& \left.-2 p_{2}^{2} J\left(\nu_{1}, \nu_{2}-1, \nu_{3}\right)+\left(-p_{1}^{2}+p_{2}^{2}+p_{3}^{2}\right) J\left(\nu_{1}, \nu_{2}, \nu_{3}-1\right)+p_{2}^{2}\left(p_{1}^{2}-p_{2}^{2}+p_{3}^{2}\right) J\left(\nu_{1}, \nu_{2}, \nu_{3}\right)\right\} \\
& C_{2}\left(\nu_{1}, \nu_{2}, \nu_{3}\right)=\frac{1}{\left(p_{3}^{2}-p_{1}^{2}-p_{2}^{2}\right)^{2}-4 p_{1}^{2} p_{2}^{2}}\left\{\left(p_{1}^{2}+p_{2}^{2}-p_{3}^{2}\right) J\left(\nu_{1}, \nu_{2}-1, \nu_{3}\right)\right. \\
& \left.\quad-2 p_{1}^{2} J\left(\nu_{1}-1, \nu_{2}, \nu_{3}\right)+\left(p_{1}^{2}-p_{2}^{2}+p_{3}^{2}\right) J\left(\nu_{1}, \nu_{2}, \nu_{3}-1\right)+p_{1}^{2}\left(-p_{1}^{2}+p_{2}^{2}+p_{3}^{2}\right) J\left(\nu_{1}, \nu_{2}, \nu_{3}\right)\right\} .
\end{aligned}
$$


Using the momentum expansion of the tensor integral defined above, we extract from Eq. (65) the relation

$$
\begin{aligned}
& \nu_{2}\left(\nu_{3}-1\right) C_{1}\left(\nu_{1}, \nu_{2}+1, \nu_{3}\right)+\nu_{2}\left(\nu_{2}+1\right)\left(C_{1}\left(\nu_{1}, \nu_{2}+2, \nu_{3}-1\right)-p_{1}^{2} C_{1}\left(\nu_{1}, \nu_{2}+2, \nu_{3}\right)\right) \\
& -\nu_{1}\left(\nu_{3}-1\right) C_{1}\left(\nu_{1}+1, \nu_{2}, \nu_{3}\right)-\nu_{1}\left(\nu_{1}+1\right)\left(C_{1}\left(\nu_{1}+2, \nu_{2}, \nu_{3}-1\right)-p_{2}^{2} C_{1}\left(\nu_{1}+2, \nu_{2}, \nu_{3}\right)\right) \\
& +\nu_{2}\left[\left(\nu_{2}+1\right)\left(J\left(\nu_{1}, \nu_{2}+2, \nu_{3}-1\right)-p_{1}^{2} J\left(\nu_{1}, \nu_{2}+2, \nu_{3}\right)\right)+\left(1+\nu_{2}+\nu_{3}-d / 2\right) J\left(\nu_{1}, \nu_{2}+1, \nu_{3}\right)\right]=0,
\end{aligned}
$$

together with the corresponding symmetric equation obtained interchanging $\left(p_{1}^{2}, \nu_{1}\right) \leftrightarrow\left(p_{2}^{2}, \nu_{2}\right)$. This result allows to express integrals in the plane $\nu_{1}+\nu_{2}+\nu_{3}=\kappa+2$ in terms of those in the two lower ones. In fact, introducing in Eq.(68) and in its symmetric one the explicit expressions for $C_{1}$ and $C_{2}$ we get

$$
J\left(\nu_{1}+2, \nu_{2}, \nu_{3}\right)=\frac{1}{\nu_{1}\left(\nu_{1}+1\right)\left(p_{1}^{2}+p_{2}^{2}-p_{3}^{2}\right) p_{2}^{2} p_{3}^{2}} \sum_{(a, b, c)} \mathcal{C}_{(a, b, c)} J\left(\nu_{1}+a, \nu_{2}+b, \nu_{3}+c\right),
$$

where the coefficients $\mathcal{C}_{(a, b, c)}$ are given by

$$
\begin{aligned}
& \mathcal{C}_{(0,0,0)}=\left(\nu_{3}-1\right)\left(\left(\nu_{1}+\nu_{2}\right) p_{1}^{2}-\nu_{2} p_{3}^{2}\right), \\
& \mathcal{C}_{(1,-1,0)}=\nu_{1}\left(\nu_{3}-1\right)\left(p_{3}^{2}-p_{1}^{2}\right), \\
& \mathcal{C}_{(-1,1,0)}=-\nu_{2}\left(\nu_{3}-1\right) p_{1}^{2}, \\
& \mathcal{C}_{(0,1,-1)}=\nu_{2}\left[\left(\nu_{2}+1\right) p_{1}^{2}-\left(2+\nu_{2}-\nu_{3}\right) p_{3}^{2}\right] \\
& \mathcal{C}_{(1,0,-1)}=\nu_{1}\left(p_{1}^{2}\left(\nu_{1}+1\right)-p_{3}^{2}\left(\nu_{3}-1\right)\right), \\
& \mathcal{C}_{(2,-1,-1)}=-\nu_{1}\left(\nu_{1}+1\right)\left(p_{1}^{2}-p_{3}^{2}\right), \\
& \mathcal{C}_{(-1,2,-1)}=-\nu_{2}\left(\nu_{2}+1\right) p_{1}^{2}, \\
& \mathcal{C}_{(2,0,-2)}=-\nu_{1}\left(\nu_{1}+1\right) p_{3}^{2}, \\
& \mathcal{C}_{(0,2,-2)}=\nu_{2}\left(\nu_{2}+1\right) p_{3}^{2},
\end{aligned}
$$




$$
\begin{aligned}
& \mathcal{C}_{(1,0,0)}= \nu_{1}\left[\left(p_{1}^{2}\right)^{2}\left(\frac{d}{2}-\nu_{1}-2\right)-p_{3}^{2}\left(p_{2}^{2}-p_{3}^{2}\right)\left(\frac{d}{2}-\nu_{1}-\nu_{3}-1\right)\right. \\
&\left.+p_{1}^{2}\left(\left(1-\frac{d}{2}\right) p_{2}^{2}+p_{3}^{2}\left(2 \nu_{1}+\nu_{3}+3-d\right)\right)\right] \\
& \mathcal{C}_{(0,1,0)}= \nu_{2} p_{1}^{2}\left[\left(1-\frac{d}{2}\right)\left(p_{1}^{2}-p_{3}^{2}\right)+\left(\frac{d}{2}-2-\nu_{2}\right) p_{2}^{2}\right] \\
& \mathcal{C}_{(0,2,-1)}=-\nu_{2}\left(\nu_{2}+1\right) p_{1}^{2} p_{3}^{2} \\
& \mathcal{C}_{(-1,2,0)}= \nu_{2}\left(\nu_{2}+1\right)\left(p_{1}^{2}\right)^{2} \\
& \mathcal{C}_{(2,0,-1)}= \nu_{1}\left(\nu_{1}+1\right) p_{3}^{2}\left(p_{1}^{2}+2 p_{2}^{2}-p_{3}^{2}\right), \\
& \mathcal{C}_{(2,-1,0)}=\nu_{1}\left(\nu_{1}+1\right) p_{2}^{2}\left(p_{1}^{2}-p_{3}^{2}\right) .
\end{aligned}
$$

Analogous results hold for $J\left(\nu_{1}, \nu_{2}+2, \nu_{3}\right)$ and $J\left(\nu_{1}, \nu_{2}, \nu_{3}+2\right)$ if we just make the usual exchanges $\left(p_{1}^{2}, \nu_{1}\right) \leftrightarrow\left(p_{2}^{2}, \nu_{2}\right)$ and $\left(p_{1}^{2}, \nu_{1}\right) \leftrightarrow\left(p_{3}^{2}, \nu_{3}\right)$ both in the integrals and in the coefficients $\mathcal{C}_{(a, b, c)}$.

\section{Conclusions}

We have shown that the solution of the conformal constraints for a scalar three-point function can be obtained directly in momentum space by solving the differential equations following from them. This has been possible having shown that these constraints take the form of a system of two PDE's of generalized hypergeometric type. The solution is expressed as a linear combination of four independent Appell's functions. The use of the momentum symmetries of the correlator allows to leave free a single multiplicative integration constant to parameterize the general solution for any conformal field theory. If this solution is compared with the position space counterpart and its Fourier representation, which is given by a family of Feynman master integrals, we obtain the explicit expression of the same integrals in terms of special functions. Our solution coincides with the one found by Boos and Davydychev using Mellin-Barnes techniques, which in our case are completely bypassed. Having established this link, we have shown that by applying special conformal constraints on the master integral representation one obtains new recursion relations.

The momentum space approach discussed in this work can be used to treat more complicated

correlators. For instance, this method can be employed in the analysis of three-point functions involving the vector and the energy momentum tensor operators, like $V V V$, TOO, TVV and TTT, as well as higher order ones, such as the scalar four-point function, whose general structure has been known for a long time [15]. Nevertheless, such a treatment is much more complicated, in the former case due to the tensor nature of the correlators, which implies a much 
more involved set of constraints, in the latter because of the increasing number of independent variables in the partial differential equations. This is left for future work.

\section{A Appendix. Invariance under the momenta parame- terization}

In this appendix we show, exploiting the invariance under rotations and dilatations, that the second of Eq.(11) is independent of which momentum is eliminated. This implies that the two different parameterizations of the special conformal constraints

$$
\begin{aligned}
& \sum_{r=1}^{n-1}\left(p_{r \mu} \frac{\partial^{2}}{\partial p_{r}^{\nu} \partial p_{r \nu}}-2 p_{r \nu} \frac{\partial^{2}}{\partial p_{r}^{\mu} \partial p_{r \nu}}+2(\right.\left.\left.\eta_{r}-d\right) \frac{\partial}{\partial p_{r}^{\mu}}+2\left(\Sigma_{\mu \nu}^{(r)}\right)_{j_{r}}^{i_{r}} \frac{\partial}{\partial p_{r \nu}}\right) \\
& \times\left\langle\mathcal{O}_{1}^{i_{1}}\left(p_{1}\right) \ldots \mathcal{O}_{r}^{j_{r}}\left(p_{r}\right) \ldots \mathcal{O}_{n}^{i_{n}}\left(p_{n}\right)\right\rangle=0 \\
& \sum_{\substack{r=1 \\
r \neq k}}^{n}\left(p_{r \mu} \frac{\partial^{2}}{\partial p_{r}^{\nu} \partial p_{r \nu}}-2 p_{r \nu} \frac{\partial^{2}}{\partial p_{r}^{\mu} \partial p_{r \nu}}+2\left(\eta_{r}-d\right) \frac{\partial}{\partial p_{r}^{\mu}}+2\left(\Sigma_{\mu \nu}^{(r)}\right)_{j_{r}}^{i_{r}} \frac{\partial}{\partial p_{r \nu}}\right) \\
& \times\left\langle\mathcal{O}_{1}^{i_{1}}\left(p_{1}\right) \ldots \mathcal{O}_{r}^{j_{r}}\left(p_{r}\right) \ldots \mathcal{O}_{n}^{i_{n}}\left(p_{n}\right)\right\rangle=0,
\end{aligned}
$$

in which we have respectively removed the dependence on $p_{n}$ and $p_{k}$ in terms of the other momenta, are indeed equivalent.

In order to simplify the presentation of the proof we introduce some convenient notations. We define

$$
\begin{aligned}
G^{i_{1} \ldots i_{n}} & \equiv\left\langle\mathcal{O}_{1}^{i_{1}}\left(p_{1}\right) \ldots \mathcal{O}_{n}^{i_{n}}\left(p_{n}\right)\right\rangle \\
\mathcal{R}_{\mu \nu}\left(p_{r}\right) & \equiv p_{r \nu} \frac{\partial}{\partial p_{r}^{\mu}}-p_{r \mu} \frac{\partial}{\partial p_{r}^{\nu}} \\
\mathcal{D}\left(p_{r}\right) & \equiv-p_{r \nu} \frac{\partial}{\partial p_{r \nu}}-d \\
\mathcal{K}_{\mu}\left(p_{r}, \eta\right) & \equiv p_{r \mu} \frac{\partial^{2}}{\partial p_{r}^{\nu} \partial p_{r \nu}}-2 p_{r \nu} \frac{\partial^{2}}{\partial p_{r}^{\mu} \partial p_{r \nu}}+2(\eta-d) \frac{\partial}{\partial p_{r}^{\mu}}
\end{aligned}
$$

and preliminarily derive two constraints, which will be used in the following, emerging from the invariance under rotations and scale transformations respectively.

Using the same procedure described in section 2, we find the constraint coming from rotational invariance

$$
\sum_{r=1}^{n-1} \mathcal{R}_{\mu \nu}\left(p_{r}\right) G^{i_{1} \ldots i_{n}}-\sum_{r=1}^{n}\left(\Sigma_{\mu \nu}^{(r)}\right)_{j_{r}}^{i_{r}} G^{i_{1} \ldots j_{r} \ldots i_{n}}=0
$$


from which, differentiating with respect to $p_{k \nu}$, we obtain

$$
\left[\sum_{r=1, r \neq k}^{n-1} \mathcal{R}_{\mu \nu}\left(p_{r}\right) \frac{\partial}{\partial p_{k \nu}}+\mathcal{F}_{\mu}\left(p_{k}\right)\right] G^{i_{1} \ldots i_{n}}-\sum_{r=1}^{n}\left(\Sigma_{\mu \nu}^{(r)}\right)_{j_{r}}^{i_{r}} \frac{\partial}{\partial p_{k \nu}} G^{i_{1} \ldots j_{r} \ldots i_{n}}=0
$$

where $\mathcal{F}_{\mu}\left(p_{k}\right)$ is defined by

$$
\mathcal{F}_{\mu}\left(p_{k}\right)=(d-1) \frac{\partial}{\partial p_{k}^{\mu}}+p_{k}^{\nu} \frac{\partial^{2}}{\partial p_{k}^{\nu} \partial p_{k}^{\mu}}-p_{k \mu} \frac{\partial^{2}}{\partial p_{k}^{\nu} \partial p_{k \nu}}
$$

Another useful relation can be obtained differentiating the dilatation constraint in the first of Eq.(111) with respect to $p_{k \mu}$

$$
\left[\sum_{r=1, r \neq k}^{n-1}\left(\mathcal{D}\left(p_{r}\right)+\eta_{r}\right) \frac{\partial}{\partial p_{k}^{\mu}}+\left(\eta_{k}+\eta_{n}-d-1\right) \frac{\partial}{\partial p_{k}^{\mu}}-p_{k}^{\nu} \frac{\partial^{2}}{\partial p_{k}^{\mu} \partial p_{k}^{\nu}}\right] G^{i_{1} \ldots i_{n}}=0 .
$$

Having derived all the necessary relations, we can proceed with the proof of equivalence between Eqs.(171) and (172). We remove from Eq.(171) the $k-t h$ term containing the spin matrix $\left(\Sigma_{\mu \nu}^{(k)}\right)_{j_{k}}^{i_{k}}$ using Eq.(75)

$$
\begin{aligned}
& {\left[\sum_{r=1}^{n-1} \mathcal{K}_{\mu}\left(p_{r}, \eta_{r}\right)+2 \sum_{r=1, r \neq k}^{n-1} \mathcal{R}_{\mu \nu}\left(p_{r}\right) \frac{\partial}{\partial p_{k \nu}}+2 \mathcal{F}_{\mu}\left(p_{k}\right)\right] G^{i_{1} \ldots i_{n}}} \\
& +2 \sum_{r=1, r \neq k}^{n-1}\left(\Sigma_{\mu \nu}^{(r)}\right)_{j_{r}}^{i_{r}}\left(\frac{\partial}{\partial p_{r \nu}}-\frac{\partial}{\partial p_{k \nu}}\right) G^{i_{1} \ldots j_{r} \ldots i_{n}}-2\left(\Sigma_{\mu \nu}^{(n)}\right)_{j_{n}}^{i_{n}} \frac{\partial}{\partial p_{k \nu}} G^{i_{1} \ldots j_{n}}=0,
\end{aligned}
$$

and then we combine the $k-t h$ operator $\mathcal{K}_{\mu}\left(p_{k}, \eta_{k}\right)$ with the $\mathcal{F}_{\mu}\left(p_{k}\right)$ contribution as

$$
\mathcal{K}_{\mu}\left(p_{k}, \eta_{k}\right)+2 \mathcal{F}_{\mu}\left(p_{k}\right)=-p_{k \mu} \frac{\partial^{2}}{\partial p_{k \nu} \partial p_{k}^{\nu}}+2\left(\eta_{k}-1\right) \frac{\partial}{\partial p_{k}^{\mu}}
$$

Using Eq.(77) we rewrite the previous equation as

$$
\mathcal{K}_{\mu}\left(p_{k}, \eta_{k}\right)+2 \mathcal{F}_{\mu}\left(p_{k}\right)=-\mathcal{K}_{\mu}\left(p_{k}, \eta_{n}\right)-2 \sum_{r=1, r \neq k}^{n-1}\left(\mathcal{D}\left(p_{r}\right)+\eta_{r}\right) \frac{\partial}{\partial p_{k}^{\mu}}
$$

so that Eq.(78) can be recast in the following form

$$
\begin{aligned}
& \left\{\sum_{r=1, r \neq k}^{n-1}\left[\mathcal{K}_{\mu}\left(p_{r}, \eta_{r}\right)+2 \mathcal{R}_{\mu \nu}\left(p_{r}\right) \frac{\partial}{\partial p_{k \nu}}-2\left(\mathcal{D}\left(p_{r}\right)+\eta_{r}\right) \frac{\partial}{\partial p_{k \mu}}\right]-\mathcal{K}_{\mu}\left(p_{k}, \eta_{n}\right)\right\} G^{i_{1} \ldots i_{n}} \\
& +2 \sum_{r=1, r \neq k}^{n-1}\left(\Sigma_{\mu \nu}^{(r)}\right)_{j_{r}}^{i_{r}}\left(\frac{\partial}{\partial p_{r \nu}}-\frac{\partial}{\partial p_{k \nu}}\right) G^{i_{1} \ldots j_{r} \ldots i_{n}}-2\left(\Sigma_{\mu \nu}^{(n)}\right)_{j_{n}}^{i_{n}} \frac{\partial}{\partial p_{k \nu}} G^{i_{1} \ldots j_{n}}=0 .
\end{aligned}
$$


In order to show the equivalence of Eq. (81) with Eq.(72) it is necessary to perform a change of variables from the independent set of momenta $\left(p_{1}, \ldots p_{n-1}\right)$ to the new independent one $\left(p_{1} \ldots p_{k-1}, p_{k+1}, \ldots p_{n}\right)$ from which $p_{k}$ has been removed using momentum conservation $p_{k}=$ $-\sum_{r=1, r \neq k}^{n} p_{r}$. In this respect all the derivatives appearing in Eq.(81) must be replaced according to

$$
\begin{array}{ll}
\frac{\partial}{\partial p_{r \mu}} \rightarrow \frac{\partial}{\partial p_{r \mu}}-\frac{\partial}{\partial p_{n \mu}} \quad \text { for } \quad r=1, \ldots n-1 \text { with } r \neq k \\
\frac{\partial}{\partial p_{k \mu}} \rightarrow-\frac{\partial}{\partial p_{n \mu}} \quad \text { for } \quad r=k .
\end{array}
$$

It is just matter of tedious algebraic manipulations to show that the operators in curly brackets in Eq.(81) simplify, after the change of variables, to give $\sum_{r=1, r \neq k}^{n} \mathcal{K}_{\mu}\left(p_{r}, \eta_{r}\right)$, while the two spin matrices sum up together and we are left with

$$
\sum_{r=1, r \neq k}^{n} \mathcal{K}_{\mu}\left(p_{r}, \eta_{r}\right) G^{i_{1}, \ldots i_{n}}+2 \sum_{r=1, r \neq k}^{n}\left(\Sigma_{\mu \nu}^{(r)}\right)_{j_{r}}^{i_{r}} \frac{\partial}{\partial p_{r \nu}} G^{i_{1} \ldots j_{r} \ldots i_{n}}=0
$$

which is exactly Eq.(72), where, now, $G^{i_{1}, \ldots i_{n}}$ is understood to be a function of the independent momenta $\left(p_{1} \ldots p_{k-1}, p_{k+1}, \ldots p_{n}\right)$. This completes our derivation proving the independence of the special conformal constraints on which momentum is removed using the momentum conservation equation.

\section{B Conformal constraints on two-point functions}

In this appendix we provide some details on the solutions of the conformal constraints for the two-point functions with conserved vector and tensor operators.

In the first case the tensor structure of the two-point function is uniquely fixed by the transversality condition $\partial^{\mu} V_{\mu}$ as

$$
G_{V}^{\alpha \beta}(p)=f\left(p^{2}\right) t^{\alpha \beta}(p), \quad \text { with } \quad t^{\alpha \beta}(p)=p^{2} \eta^{\alpha \beta}-p^{\alpha} p^{\beta} .
$$

For the sake of simplicity, we have employed in the previous equation a slightly different notation with respect to Eq.(17), which, anyway, can be recovered with the identification $f\left(p^{2}\right)=f_{V}\left(p^{2}\right) / p^{2}$.

In order to exploit the invariance under scale and special conformal transformations it is useful to compute first and second order derivatives of the $t^{\alpha \beta}$ tensor structure. In particular we have

$$
\begin{aligned}
t_{1}^{\alpha \beta, \mu}(p) & \equiv \frac{\partial}{\partial p_{\mu}} t^{\alpha \beta}(p)=2 p^{\mu} \eta^{\alpha \beta}-p^{\alpha} \eta^{\mu \beta}-p^{\beta} \eta^{\mu \alpha}, \\
t_{2}^{\alpha \beta, \mu \nu}(p) & \equiv \frac{\partial^{2}}{\partial p_{\mu} \partial p_{\nu}} t^{\alpha \beta}(p)=2 \eta^{\mu \nu} \eta^{\alpha \beta}-\eta^{\nu \alpha} \eta^{\mu \beta}-\eta^{\nu \beta} \eta^{\mu \alpha},
\end{aligned}
$$


with the properties

$$
\begin{array}{ll}
p_{\mu} t_{1}^{\alpha \beta, \mu}(p)=2 t^{\alpha \beta}(p), & t_{1}^{\alpha \beta, \alpha}(p)=-(d-1) p^{\beta}, \\
p_{\mu} t_{2}^{\alpha \beta, \mu \nu}(p)=t_{1}^{\alpha \beta, \nu}(p), & t_{2}^{\alpha \beta, \mu \mu}(p)=2(d-1) \eta^{\alpha \beta} .
\end{array}
$$

As we have already mentioned, the invariance under scale transformations implies

$$
f\left(p^{2}\right)=\left(p^{2}\right)^{\lambda} \quad \text { with } \quad \lambda=\frac{\eta_{1}+\eta_{2}-d}{2}-1,
$$

which can be easily derived from the first order differential equation in (12) using Eq.(85). Having determined the structure of the scalar function $f\left(p^{2}\right)$, one can compute the derivatives appearing in the second of Eq.(12), namely the constraint following from the invariance under the special conformal transformations

$$
\begin{aligned}
& \frac{\partial}{\partial p_{\mu}} G_{V}^{\alpha \beta}(p)=\left(p^{2}\right)^{\lambda-1}\left[2 \lambda p^{\mu} t^{\alpha \beta}(p)+p^{2} t_{1}^{\alpha \beta, \mu}(p)\right] \\
& \frac{\partial^{2}}{\partial p_{\mu} \partial p_{\nu}} G_{V}^{\alpha \beta}(p)=\left(p^{2}\right)^{\lambda-2}\left[4 \lambda(\lambda-1) p^{\mu} p^{\nu} t^{\alpha \beta}(p)+2 \lambda p^{2} \eta^{\mu \nu} t^{\alpha \beta}(p)+2 \lambda p^{2} p^{\mu} t_{1}^{\alpha \beta, \nu}(p)\right. \\
&\left.+2 \lambda p^{2} p^{\nu} t_{1}^{\alpha \beta, \mu}(p)+\left(p^{2}\right)^{2} t_{2}^{\alpha \beta, \mu \nu}(p)\right]
\end{aligned}
$$

where the definitions in Eq.(85) have been used. Concerning the spin dependent part in Eq.(12), we use the spin matrix for the vector field, which, in our conventions, reads as

$$
\left(\Sigma_{\mu \nu}^{(V)}\right)_{\beta}^{\alpha}=\delta_{\mu}^{\alpha} \eta_{\nu \beta}-\delta_{\nu}^{\alpha} \eta_{\mu \beta},
$$

and obtain

$$
2\left(\sum_{\mu \nu}^{(V)}\right)_{\rho}^{\alpha} \frac{\partial}{\partial p_{\nu}} G_{V}^{\rho \beta}(p)=-\left(p^{2}\right)^{\lambda-1}\left[2 \lambda p^{\alpha} t_{\mu}^{\beta}(p)+(d-1) p^{2} p^{\beta} \delta_{\mu}^{\alpha}+p^{2} t_{1 \mu}^{\beta, \alpha}(p)\right] .
$$

Employing the results derived in Eq.(88) and Eq.(90), we have fully determined the special conformal constraint on the two-point vector function. Then we can project the second of Eq.(12) onto the three independent tensor structures $p_{\mu} \eta_{\alpha \beta}, p_{\alpha} \eta_{\mu \beta}, p_{\beta} \eta_{\alpha \mu}$, and setting $\lambda$ to the value given in Eq.(87), we finally obtain three equations for the scale dimensions $\eta_{i}$ of the vector operators

$$
\left\{\begin{array}{l}
\left(\eta_{1}-\eta_{2}\right)\left(\eta_{1}+\eta_{2}-d\right)=0 \\
\eta_{1}-d+1=0 \\
\eta_{2}-d+1=0
\end{array}\right.
$$


The previous system of equations can be consistently solved only for $\eta_{1}=\eta_{2}=d-1$, as expected. This completes our derivation of the vector two-point function which, up to an arbitrary multiplicative constant, can be written as in Eq.(18).

The characterization of the two-point function with a symmetric, traceless and conserved rank-2 tensor follows the same lines of reasoning already explained in the vector case. These conditions (see Eq.(19)) fix completely the tensor structure of the two-point function as

$$
G_{T}^{\alpha \beta \mu \nu}(p)=g\left(p^{2}\right) T^{\alpha \beta \mu \nu}(p)
$$

with

$$
T^{\alpha \beta \mu \nu}(p)=\frac{1}{2}\left[t^{\alpha \mu}(p) t^{\beta \nu}(p)+t^{\alpha \nu}(p) t^{\beta \mu}(p)\right]-\frac{1}{d-1} t^{\alpha \beta}(p) t^{\mu \nu}(p) .
$$

In order to recover the convention used in section [3, notice that $g\left(p^{2}\right)=f_{T}\left(p^{2}\right) /\left(p^{2}\right)^{2}$.

As in the previous case, we give the first and second order derivatives of the $T^{\alpha \beta \mu \nu}(p)$ tensor structure

$$
\begin{aligned}
T_{1}^{\alpha \beta \mu \nu, \rho}(p) \equiv & \frac{\partial}{\partial p_{\rho}} T^{\alpha \beta \mu \nu}(p)=\frac{1}{2}\left[t_{1}^{\alpha \mu, \rho}(p) t^{\beta \nu}(p)+t^{\alpha \mu}(p) t_{1}^{\beta \nu, \rho}(p)+(\mu \leftrightarrow \nu)\right] \\
& -\frac{1}{d-1}\left[t_{1}^{\alpha \beta, \rho}(p) t^{\mu \nu}(p)+t^{\alpha \beta}(p) t_{1}^{\mu \nu, \rho}(p)\right] \\
T_{2}^{\alpha \beta \mu \nu, \rho \sigma}(p) \equiv & \frac{\partial}{\partial p_{\rho} \partial p_{\sigma}} T^{\alpha \beta \mu \nu}(p)=\frac{1}{2}\left[t_{2}^{\alpha \mu, \rho \sigma}(p) t^{\beta \nu}(p)+t_{1}^{\alpha \mu, \rho}(p) t_{1}^{\beta \nu, \sigma}(p)+t_{1}^{\alpha \mu, \sigma}(p) t_{1}^{\beta \nu, \rho}(p)\right. \\
& \left.+t^{\alpha \mu}(p) t_{2}^{\beta \nu, \rho \sigma}(p)+(\mu \leftrightarrow \nu)\right] \\
& -\frac{1}{d-1}\left[t_{2}^{\alpha \beta, \rho \sigma}(p) t^{\mu \nu}(p)+t_{1}^{\alpha \beta, \rho}(p) t_{1}^{\mu \nu, \sigma}(p)+(\mu \nu) \leftrightarrow(\alpha \beta)\right]
\end{aligned}
$$

together with some of their properties

$$
p_{\rho} T_{1}^{\alpha \beta \mu \nu, \rho}(p)=4 T^{\alpha \beta \mu \nu}(p), \quad p_{\rho} T_{2}^{\alpha \beta \mu \nu, \rho \sigma}(p)=3 T_{1}^{\alpha \beta \mu \nu, \sigma}(p) .
$$

As we have already stressed, the first of Eq.(12) defines the scaling behavior of the two-point function, providing, therefore, the functional form of $g\left(p^{2}\right)$ which is given by

$$
g\left(p^{2}\right)=\left(p^{2}\right)^{\lambda} \quad \text { with } \quad \lambda=\frac{\eta_{1}+\eta_{2}-d}{2}-2 .
$$

On the other hand, the second of Eq.(12), namely the constraint from the special conformal transformations, fixes the scaling dimensions of the tensor operators. In this case the spin connection is given by

$$
\left(\Sigma_{\mu \nu}^{(T)}\right)_{\rho \sigma}^{\alpha \beta}=\left(\delta_{\mu}^{\alpha} \eta_{\nu \rho}-\delta_{\nu}^{\alpha} \eta_{\mu \rho}\right) \delta_{\sigma}^{\beta}+\left(\delta_{\mu}^{\beta} \eta_{\nu \sigma}-\delta_{\nu}^{\beta} \eta_{\mu \sigma}\right) \delta_{\rho}^{\alpha}
$$


The algebra is straightforward but rather cumbersome due to the proliferation of indices. Here we give only the final result, which can be obtained projecting Eq.(12), making use of Eq.(94), onto the different independent tensor structures

$$
\left\{\begin{array}{l}
\left(\eta_{1}-\eta_{2}\right)\left(\eta_{1}+\eta_{2}-d\right)=0 \\
\eta_{1}-d=0 \\
\eta_{2}-d=0
\end{array}\right.
$$

which implies the solution $\eta_{1}=\eta_{2}=d$, as described in Eq.(21).

\section{References}

[1] H. Osborn and A. C. Petkou, Ann. Phys. 231, 311 (1994), arXiv:hep-th/9307010.

[2] J. Erdmenger and H. Osborn, Nucl.Phys. B483, 431 (1997), arXiv:hep-th/0103237.

[3] C. Corianò, L. Delle Rose, E. Mottola, and M. Serino, JHEP 1208, 147 (2012), arXiv:1203.1339,

[4] M. Giannotti and E. Mottola, Phys. Rev. D79, 045014 (2009), arXiv:0812.0351.

[5] E. Mottola, Acta Physica Polonica B41, 2031 (2010), arXiv:1008.5006.

[6] R. Armillis, C. Corianò, and L. Delle Rose, Phys. Rev. D81, 085001 (2010), arXiv:0910.3381.

[7] R. Armillis, C. Corianò, and L. Delle Rose, Phys. Lett. B682, 322 (2009), arXiv:0909.4522.

[8] R. Armillis, C. Corianò, and L. Delle Rose, Phys.Rev. D82, 064023 (2010), arXiv:1005.4173.

[9] C. Corianò, L. Delle Rose, A. Quintavalle, and M. Serino, Phys.Lett. B700, 29 (2011), arXiv:1101.1624,

[10] C. Corianò, L. Delle Rose, and M. Serino, Phys.Rev. D83, 125028 (2011), arXiv:1102.4558.

[11] I. Antoniadis, P. O. Mazur, and E. Mottola, Phys. Rev. Lett. D79, 14 (1997), astro-ph/9611208.

[12] I. Antoniadis, P. O. Mazur, and E. Mottola, JCAP 09, 024084 (2012), arXiv:1103.4164. 
[13] J. M. Maldacena and G. L. Pimentel, JHEP 1109, 045 (2011), arXiv:1104.2846.

[14] A. Kehagias and A. Riotto, Nucl.Phys. B864, 492 (2012), arXiv:1205.1523.

[15] A. M. Polyakov, JETP Lett. 12, 381 (1970).

[16] A. Cappelli, D. Friedan, and J. Latorre, Nucl.Phys. B352, 616 (1991).

[17] L. S. Brown and J. C. Collins, Ann. Phys. 130, 215 (1980).

[18] M. J. Duff, Nucl. Phys. B125, 334 (1977).

[19] P. R. Anderson, C. Molina-Paris, and E. Mottola, Phys. Rev. D67, 024026 (2003), 0209075.

[20] P. Appell and J. Kampé de Fériet, Fonctions hypergéométriques et hypersphériques (Gauthier-Villars, 1926).

[21] W. N. Bailey, Generalized hypergeometric series (Cambridge University Press, 1935).

[22] L. J. Slater, Generalized hypergeometric functions (Cambridge University Press, 1966).

[23] E. Boos and A. I. Davydychev, Moscow Univ.Phys.Bull. 42N3, 6 (1987).

[24] A. I. Davydychev, J.Phys.A A25, 5587 (1992). 